\title{
La première maison d'Amazonie. Le Formatif dans la province de Pastaza, Équateur
}

The first house in Amazonia. The Formative in the Pastaza province

La primera casa en la Amazonía. El Formativo en la provincia de Pastaza

\section{Stéphen Rostain et Geoffroy de Saulieu}

\section{OpenEdition}

\section{Journals}

Édition électronique

URL : https://journals.openedition.org/jsa/14282

DOI : $10.4000 /$ jsa. 14282

ISSN : $1957-7842$

\section{Éditeur}

Société des américanistes

\section{Édition imprimée}

Date de publication : 31 décembre 2015

Pagination : 51-81

ISSN : 0037-9174

\section{Référence électronique}

Stéphen Rostain et Geoffroy de Saulieu, «La première maison d'Amazonie. Le Formatif dans la province de Pastaza, Équateur », Journal de la Société des américanistes [En ligne], 101-1 et 2 | 2015, mis en ligne le 15 mars 2016, consulté le 24 février 2023. URL : http://journals.openedition.org/jsa/ 14282 ; DOI : https://doi.org/10.4000/jsa.14282 


\title{
La première maison d'Amazonie. Le Formatif dans la province de Pastaza, Équateur
}

\author{
Stéphen Rostain* et Geoffroy de Saulieu**
}

Le projet interdisciplinaire «Zulay, le portail précolombien de l'Amazonie » dans la vallée du haut Pastaza, en Amazonie équatorienne, a permis la découverte et la fouille horizontale extensive des restes d'une maison du Formatif tardif, datée de 1496-1302 av. J.-C. calibrée. L'étude de la répartition des trous de poteau, des fosses et du foyer empierré conduit à reconstituer un plan d'habitat comparable à celui des cases amérindiennes actuelles de la région. En outre, une extrémité de poteau de bois s'était parfaitement conservée dans sa matrice au niveau de la nappe phréatique. Il était fait d'un tronc d'arbre élagué et placé à l'envers dans le sol, la cime vers le bas. La rareté de structures domestiques précolombiennes étudiées dans la région rend cette trouvaille de la plus ancienne maison d'Amazonie particulièrement remarquable. [Mots-clés: Formatif, Amazonie, Équateur, maison, trou de poteau, céramique.]

The first house in Amazonia. The Formative in the Pastaza province. The interdisciplinary project entitled «Zulay, the pre-Columbian portal to the Amazon », in the upper valley of the Pastaza (Ecuadorian Amazon), has led to the discovery and extensive excavation of the remains of a late Formative house, dated cal. 1496$1302 \mathrm{BC}$. The study of the distribution of postholes, pits, and stone-made hearth has enabled archaeologists to reconstruct a dwelling pattern similar to that of present indigenous houses in the area. In addition, one end of a perfectly preserved wooden post has been found at an undisturbed cross section at groundwater level. It consists of a trimmed tree trunk, which was placed upside down in the ground, the crown towards the earth. Due to the scarcity of pre-Columbian domestic structures that have been studied in the region, the discovery of one of the most ancient Amazonian houses constitutes a remarkable finding. [Key words: Formative, Amazonia, Ecuador, house, posthole, pottery.]

La primera casa en la Amazonía. El Formativo en la provincia de Pastaza. El proyecto interdisciplinario «Zulay, el pórtico precolombino de la Amazonía » en

* CNRS, UMR 8096 ArchAm, Maison archéologie et ethnologie, René-Ginouvès, 21, allée de l'université, 92023 Nanterre CEDEX [stephen.rostain@mae.u-paris10.fr].

** IRD, UMR 208 Paloc, Yaoundé [geoffroy.desaulieu@ird.fr]. 
el valle del Alto Pastaza, en Amazonía ecuatoriana, dio lugar al descubrimiento y a la excavación horizontal extensiva de los restos de una casa del Formativo tardío, con fecha calibrada 1496-1302 a.C. El estudio de la distribución de los hoyos de postes, las fosas y el fogón empedrado permitió la reconstitución de un plano de hábitat comparable con aquel de las actuales casas amerindias de la región. Además, un extremo de poste de madera se había conservado perfectamente en su matríz al nivel de la capa freática. Éste había sido elaborado a partir del tronco de un árbol podado y colocado al revés, con la copa hacia abajo. La carencia de estructuras domésticas precolombinas estudiadas en la región hace de este hallazgo de la casa de Amazonía más antigua, algo particularmente notable. [Palabras clave: Formativo, Amazonía, Ecuador, casa, hoyo de poste, cerámica.]

«Ici l'espace est tout, car le temps n'anime plus la mémoire. » Gaston Bachelard, « La poétique de l'espace », 1957.

\section{Introduction}

Les développements réalisés par l'archéologie amazonienne ont été tout autant fulgurants que spectaculaires depuis deux décennies. Le paradigme dominant autrefois, qui reposait pour une large part sur des préconçus et de l'ethno-comparatisme direct, adoptait un déterminisme environnemental pour nier la possibilité de grands établissements humains ou d'innovations culturelles marquantes à cause d'un milieu tropical jugé défavorable, pauvre, voire destructeur pour l'homme. La thèse était osée mais, comme toutes les thèses, parfaitement défendable. Ainsi, nombreux furent ceux qui suivirent ce courant de pensée jusqu'à ce que les nouvelles évidences archéologiques le rendent inacceptable. Il a alors été rejeté par un nombre croissant de chercheurs pour être remplacé par des approches adaptées dans lesquelles se sont progressivement imposés les principes de l'écologie historique. En s'appuyant sur de réelles données de terrain, qui démontrent notamment à quel point les environnements amazoniens ont été modifiés par l'homme dans le passé, l'écologie historique offre un chemin de compréhension aujourd'hui amplement adopté par les archéologues. Libérée des contraintes théoriques antérieures, la discipline connut des avancées remarquables, notamment dans l'étude des sols anthropiques, des terrassements, de la domestication et de l'utilisation des ressources végétales, de la dispersion des populations et des cultures, de la chronologie ou des activités cérémonielles passées.

Toutefois, la roue du progrès semble avoir quelque peu oublié l'étude de l'habitat même des premiers hommes d'Amazonie. Malgré les efforts évidents développés par l'ethnohistoire dans l'analyse des conditions de vie et d'occupation des groupes autochtones, la fouille et l'observation de la maison précolombienne demeurent limitées. L'examen synchronique des fonds de 
cabane est exceptionnel, laissant un vide dans la recherche et de nombreuses interrogations en suspens sur le quotidien des anciens occupants.

Un coin du voile vient pourtant d'être récemment levé en Équateur grâce au programme interdisciplinaire et international «Zulay, le portail précolombien de l'Amazonie », mené par les auteurs de 2011 à 2014 dans le bassin du haut Pastaza (Rostain et Saulieu 2014).

Cette recherche partait du présupposé que la vallée du Pastaza, l'une des rares descendant en ligne droite d'ouest en est des Andes équatoriennes vers 1'Amazonie (Figure 1), avait joué un rôle important dans les échanges entre sociétés pacifiques, andines et amazoniennes, durant l'époque précolombienne. De là, on pouvait imaginer que des développements culturels notables et anciens avaient vu le jour dans cette région.

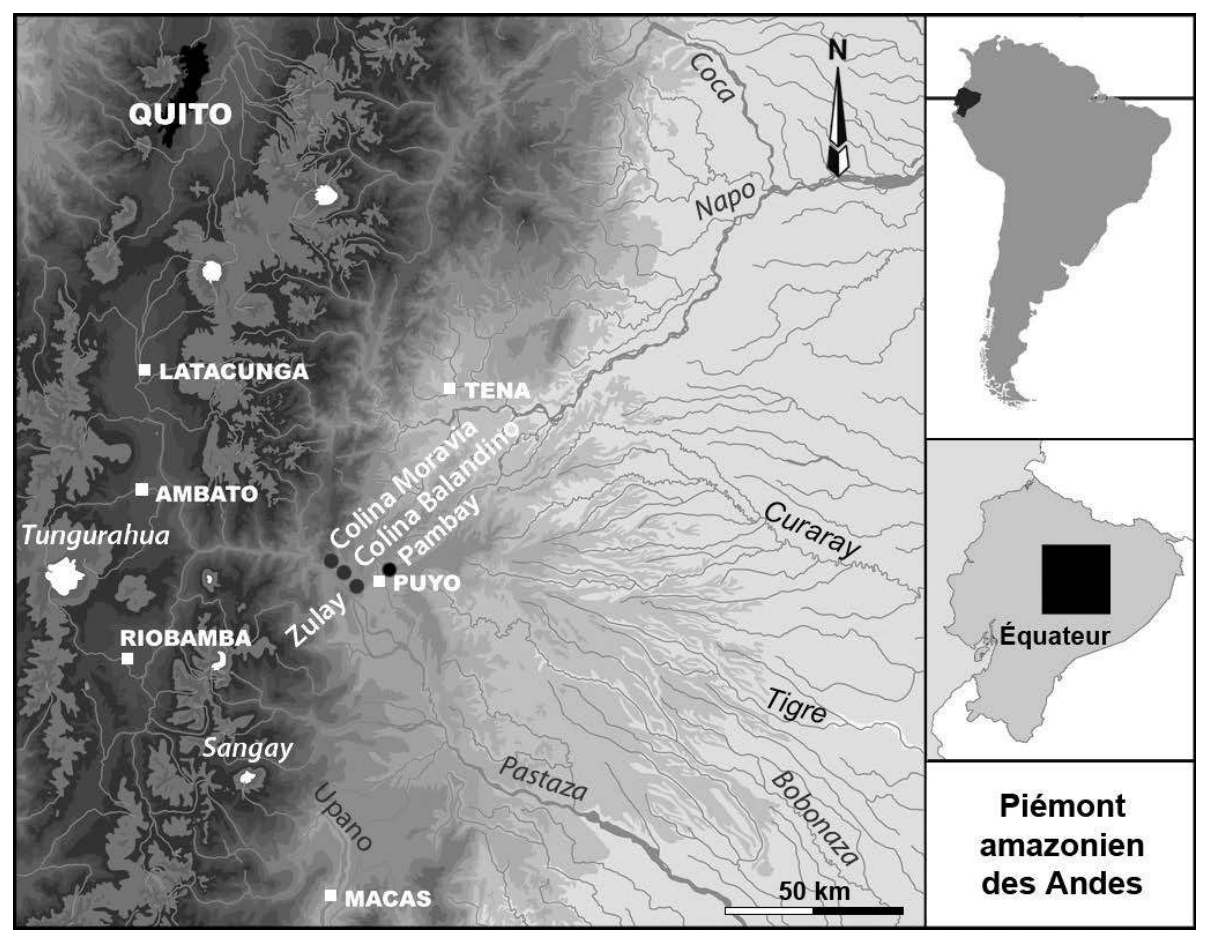

Fig. 1 - Localisation de l'aire de recherche du programme « Zulay » avec les principaux sites étudiés indiqués en diagonale (fond de carte de L. Billault).

Il faut savoir que la rivière Pastaza naît à la confluence des rios Chambo et Patate, à environ 1900 m d'altitude, au pied du volcan Tungurahua, situé sur le versant oriental des Andes équatoriennes. La rivière descend ensuite un canyon vertigineux, ponctué de cascades et de chaos rocheux qui débouche 
brutalement à environ 1200 m d'altitude sur une forêt tropicale hyper humide (Figure 2). Cette aire, formée de plateaux et de collines, s'étend sur 30 à $40 \mathrm{~km}$ vers l'est, puis un palier s'abaisse brusquement à $600 \mathrm{~m}$. Il s'agit en réalité du gigantesque cône de déjection du Pastaza qui a charrié pendant des millénaires les sédiments andins et les rejets des volcans de la Cordillère orientale, notamment du puissant Tungurahua (Saulieu et al. 2014). C'est sur cette sorte de marche que l'on trouve les villes pionnières de Mera, Shell et Puyo. Leur fondation assez récente rappelle que cette région a souvent été considérée comme une marge, impropre à la civilisation moderne.

Son accès, longtemps resté difficile et périlleux, et son climat excessivement humide expliquent également en partie le désintérêt dont elle a été l'objet de la part des archéologues. Rappelons néanmoins que ces derniers ont été, dès les années 1960, absorbés par les Hautes Terres et surtout la côte pacifique où l'on commençait à découvrir les vestiges impressionnants des cultures précolombiennes du Formatif équatorien (3500-300 av. J.-C.). C'est pour cela que, si la connaissance de cette période s'est fortement développée à l'ouest des Andes, où l'on peut même reconstituer le plan des unités domestiques, on ne peut en dire autant pour l'est des Andes.

L'Amazonie équatorienne a d'abord été mise en valeur assez tardivement dans les années 1970-1980 par les travaux du Père Pedro Porras. En dépit de son apport indéniable par la révélation au grand public de la richesse archéologique de cette région des Basses Terres tropicales, cet archéologue amateur n'a hélas pas toujours suivi une démarche scientifique rigoureuse, ce qui invalide certains de ses résultats. L'un de ses ouvrages (Porras 1987) a notamment été consacré à la vallée de l'Upano, située à $80 \mathrm{~km}$ au sud de Puyo, où des monticules rectangulaires ont été construits par des populations précolombiennes le long du piémont durant les derniers siècles avant notre ère. L'étude de cette région a été reprise dans les années 1990 avec le projet « Sangay-Upano », dirigé par Stéphen Rostain et Ernesto Salazar, puis avec le projet « Río Blanco » mené par Stéphen Rostain (Rostain 1999, 2012). Ces nouvelles recherches ont, entre autres, permis de reconstituer une chronologie culturelle qui montre que, si l'architecture monumentale de cette région d'Amazonie est postérieure au Formatif (Figure 3, page suivante), une occupation vraiment formative y est clairement attestée avec la culture Sangay (Rostain 2010). En outre, Sangay est l'un des rares sites d'Amazonie où a été mis en évidence un sol de maison avec toute une série de traces et de vestiges très informatifs (Rostain 2011).

Plus au sud encore, les travaux initiés par Jean Guffroy dans la province de Zamora-Chinchipe au début des années 2000 ont révélé l'existence d'un site formatif ancien, datée autour de 2800-2500 avant notre ère (Valdez et al. 2005). Santa-Ana/La Florida était un centre cérémoniel composé d'une structure arrondie de pierres, construite de paliers concentriques, vraisemblablement successifs. Des vestiges de très haute qualité y ont été trouvés dans des caches 

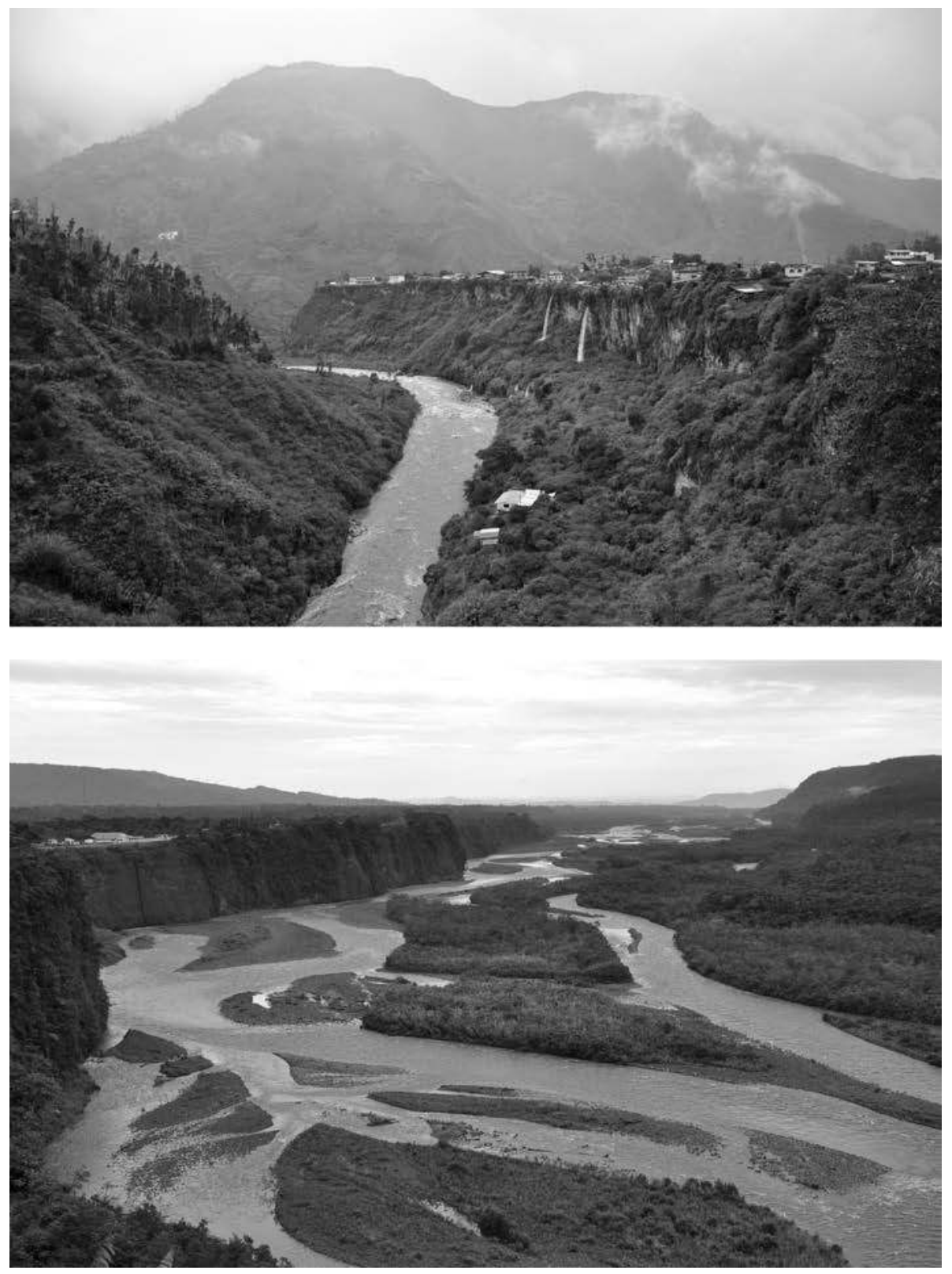

Fig. 2 - Canyon du haut Pastaza dans les Andes, au niveau de Baños, et débouché de la gorge dans la plaine amazonienne à l'est, avant Puyo (photographies S. Rostain). 


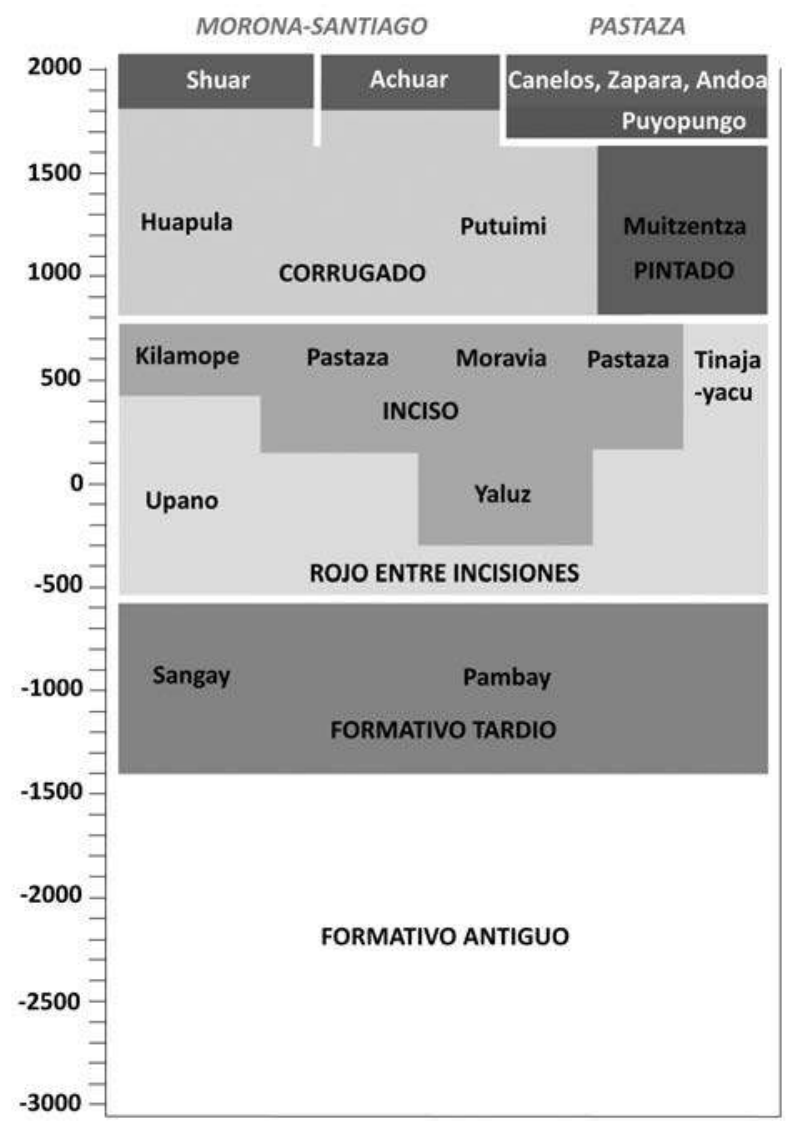

Fig. 3 - Chronologie simplifiée des vallées de l'Upano et du Pastaza, Amazonie équatorienne (dessin S. Rostain et G. de Saulieu).

et des sépultures datées de plus de 5000 ans : bouteilles de céramique à anse en étrier, bols de pierre sculptée, pendentifs et perles de pierre semi-précieuse, etc. La délicatesse et 1'élaboration des décors zoomorphes représentés sur ces pièces sont stupéfiantes, suggérant un art abouti. Ce dernier présente des similitudes remarquables avec le style développé à Chavin, une autre implantation architecturale des flancs orientaux des Andes plus au sud, mais ultérieure de plusieurs siècles. Cela soulève d'ailleurs une des interrogations relatives au site de Santa-Ana/La Florida, puisque tant sa localisation que les traits culturels qu'il expose laissent penser bien plus à un établissement d'origine andine que d'influence amazonienne. Il est ainsi difficile de qualifier le complexe d'amazonien car, en premier lieu, il se situe dans une haute vallée andine très encaissée, dont la végétation est tropicale, avant de s'assécher rapidement 
jusqu'à sa confluence avec le Marañón au Pérou, situé à plus de $100 \mathrm{~km}$ au sud, à proximité de Jaen, dans la région de Cajamarca. Son seul lien avec la plaine amazonienne se situe donc assez loin, au Pérou, après que le Marañón a franchi une série de pongos (rapides) dans la province péruvienne d'Amazonas. La seconde raison de la réticence d'une attribution réellement amazonienne est que son art mobilier et son architecture en pierre le rattachent plutôt au monde andin contemporain de l'époque : il ne dépare pas dans le paysage culturel des périodes précéramique et initiale de la Sierra et de la côte péruviennes, tout en montrant des liens avec le Formatif de la côte équatorienne. En tout cas, outre son architecture complexe et ses vestiges raffinés, la découverte la plus proéminente de Santa-Ana/La Florida demeure sans nul doute la preuve de la domestication et la consommation du cacao (Theobroma sp.) quelque 1500 ans avant la Mésoamérique (Zarillo 2012).

De tous les sites archéologiques découverts durant nos investigations dans le bassin du Pastaza, Pambay est l'un des plus remarquables car il a permis la mise en évidence, sur un sommet de colline, d'une phase unique d'occupation caractérisée par des trous de poteaux et autres anomalies, ce qui rend possible la restitution du plan d'une maison de cette haute époque. Cette découverte est exceptionnelle car, au faible nombre des recherches réalisées en Amazonie, il faut ajouter le fait que l'on rencontre généralement des sites à occupations multiples qui se traduisent par le pullulement des trous de poteaux rendant la reconstitution d'un plan souvent totalement illusoire.

Cet article présente donc le plan de la plus ancienne maison d'Amazonie connue à ce jour et ce qu'il nous apprend sur le Formatif de cette portion de forêt tropicale.

\section{Une maison vieille de 3000 ans à Pambay}

Parmi les sites repérés dans le cadre du projet «Zulay », deux possèdent une occupation formative avec des datations voisines. Colline Moravia, un établissement à multiples occupations situé sur une élévation d'origine volcanique à proximité de Shell, a fourni deux datations (Figure 4, page suivante).

Le site de Pambay a, quant à lui, été décelé dans le profil d'un chemin récemment creusé à travers une colline (1 $1^{\circ} 28^{\prime} 10.94$ ' ' $\left.5 \mathrm{~S}, 78^{\circ} 00^{\prime} 07.82^{\prime \prime} 0 \mathrm{~W}\right)$, à $940 \mathrm{~m}$ d'altitude au-dessus du niveau de la mer, près de la ville de Puyo (Figure 5, page suivante), accessible par le contournement routier nord de la ville. Dans la paroi longeant le chemin, à peu près à $100 \mathrm{~cm}$ de profondeur, se distinguait un foyer empierré de $170 \mathrm{~cm}$ de diamètre pour $30 \mathrm{~cm}$ d'épaisseur (Figure 6 , pages suivantes). Le charbon extrait de cette structure a fourni une datation calibrée de 1496-1302 av. J.-C. (Figure 4, page suivante). 


\begin{tabular}{|c|c|c|c|}
\hline No de laboratoire & $\begin{array}{c}\text { Datation }{ }^{14} \mathbf{C} \\
\text { non calibrée }\end{array}$ & $\begin{array}{c}\text { Datation calibrée } \\
\text { à } \mathbf{2} \boldsymbol{\sigma} \\
(\text { OxCal.v4.2 .4) }\end{array}$ & Site \\
\hline Beta-324360 & $3460 \pm 30$ ans AP & $1881-1692$ av. J.-C. & Colline Moravia \\
\hline UBA-20797 & $3671 \pm 32$ ans AP & $2141-1950$ av. J.-C. & Colline Moravia \\
\hline Lyon-9521 & $3135 \pm 30$ ans AP & $1496-1302$ av. J.-C. & Pambay \\
\hline
\end{tabular}

Fig. 4 - Datation de charbons de bois des contextes Pambay du Pastaza.

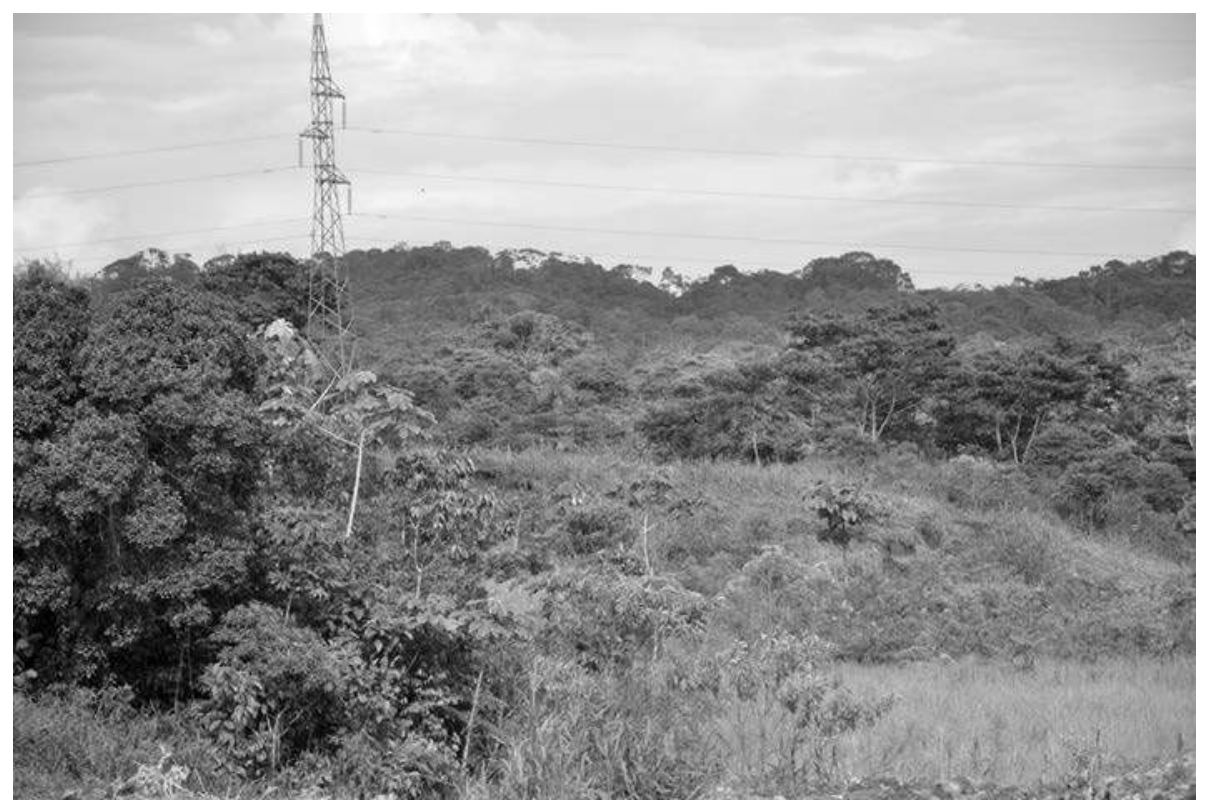

Fig. 5 - Petite colline arrondie au sommet de laquelle était implanté le site formatif de Pambay (photographie S. Rostain).

Le site se trouve au sommet d'une colline au modelé arrondi, bordée du sud-est au sud-ouest par des bas-fonds humides et deux petits ruisseaux, contribuant au cours d'eau Pambay qui se connecte non loin de là à la rivière Puyo, un affluent du Pastaza.

L'étude de la coupe stratigraphique a pris le foyer pour repère de base. Lors du nettoyage de la paroi, il est apparu que la partie nord-est, contrairement à la partie sud-ouest, laissait voir des irrégularités intéressantes. Aussi avons-nous décidé de réaliser le relevé du segment nord-est sur une longueur de $10 \mathrm{~m}$. Les différentes couches et la position stratigraphique du foyer montrent que, malgré la situation du site en sommet de colline, une succession de dépôts sédimentaires est intervenue depuis l'abandon de la maison (Figure 7) : sous une couche de 




Fig. 6 - Dégagement du foyer charbonneux de Pambay (en sombre) dans la coupe du chemin (photographie S. Rostain).

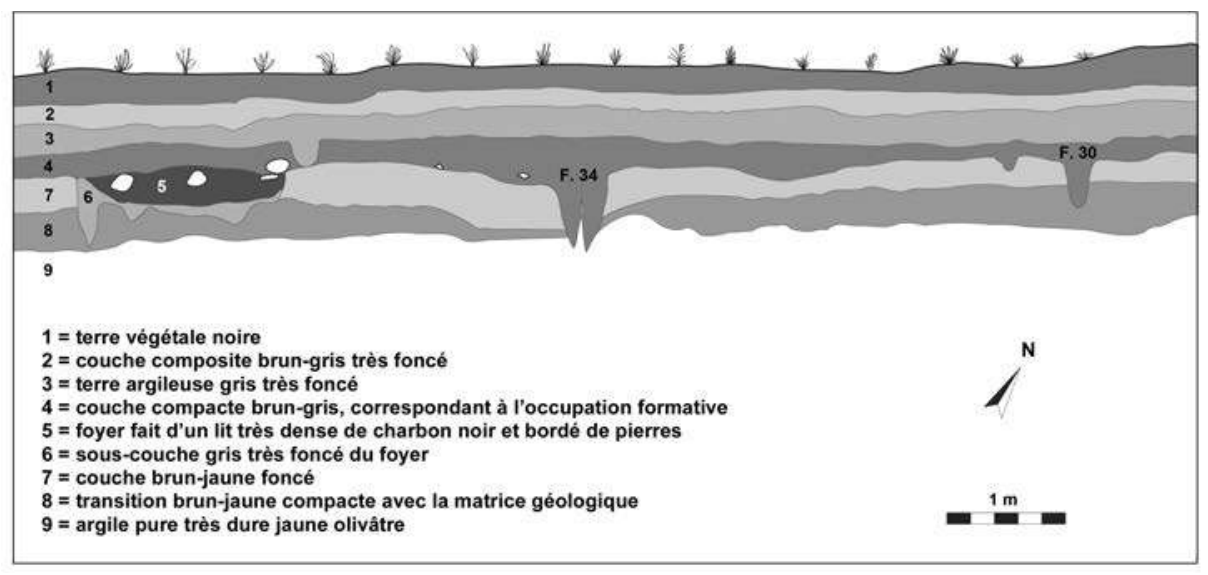

Fig. 7 - Stratigraphie du site de Pambay le long de la coupe nord du chemin, avec le foyer construit en pierres (indiquées en blanc) (dessin G. de Saulieu).

terre végétale noire (Munsell 10YR2/1) organique avec beaucoup de racines (1), on trouve une couche brun-gris très foncé (Munsell 10YR3/2), de nature composite, mélange d'argile, de charbon et de racines (2). Elle se révèle par contraste comme nettement plus claire que la précédente et que la suivante. 
Cette dernière (3), une couche gris très foncé (Munsell 10YR3/1) argileuse, présente beaucoup de charbon et de racines, ce qui rappelle la couche 1, malgré une tendance plus argileuse et moins organique. La quatrième couche, brungris (Munsell 10YR3/2 à 10YR3/3) argileuse avec des racines (elle tire sur le jaunâtre en s'éloignant du chemin), présente des fragments charbonneux, mais en nette diminution par rapport aux couches supérieures. En outre, la terre y est nettement plus compacte. On remarque quelques petites pierres chauffées (roses à rouges) de manière dispersée. Ce niveau correspond à l'occupation où le foyer, visible en coupe, était en activité. Cette couche apparaît à une profondeur de 65 à $90 \mathrm{~cm}$ depuis la surface du sol en fonction de la topographie de surface. Le foyer se décompose en un lit très dense de charbon noir (Munsell 2YR2/0) bordé de pierres (5), et d'une sous-couche gris très foncé (Munsell 10YR3/1) argilo-cendreuse avec des morceaux de charbon (6). Dessous, une couche (7) brun-jaune foncé (Munsell 10YR4/4), de nature argileuse, comporte quelques charbons. Des infiltrations tubulaires de sédiment blanc compact, probablement des cendres volcaniques, ont été repérées. Ce matériau a apparemment pénétré des galeries de vers de terre ou d'insectes sociaux, dans lesquelles il a été piégé. Commence ensuite la transition avec la matrice géologique ( 8 et 9) : une couche brun-jaune (Munsell 10YR5/8), compacte, homogène, avec des charbons rares et des taches rougeâtres, caractéristiques de phénomènes hydromorphes, fait ensuite place à une couche argileuse pure, de couleur jaune olivâtre (2YR6/4), très compacte, dure et imperméable.

De manière générale il faut remarquer que, comme dans d'autres sites de la région, les couches scellant le gisement en surface montrent une alternance de tons : couche $n^{\circ} 1$ sombre, couche $n^{\circ} 2$ claire, couche $n^{\circ} 3$ sombre. Ce phénomène pourrait être lié à des dépôts de cendres volcaniques, ce qui expliquerait l'étrange sédimentation du site : en effet, bien que le site se trouve en sommet de colline, un dépôt sédimentaire épais s'est mis en place postérieurement à l'occupation formative en dépit du régime d'intenses précipitations que connaît la région. Sans doute faut-il sur ce point insister sur le rôle de fixateur de sédiments qu'a joué la végétation tropicale.

L'occupation humaine est visible à divers niveaux. Dans les couches 1 et 2 , le matériel céramique est peu dense, dispersé et relativement grossier (poterie à paroi épaisse de plus de $0,5 \mathrm{~cm}$, pâte hétérogène, peu ou pas de décor). Il s'agit de l'occupation tardive, diffuse dans la région, que nous attribuons à la culture Putuimi (Rostain et al. 2014). Si les prospections de 2011 nous avaient permis de trouver un matériel fin plus ancien dans les remblais des machines, attribuable à la culture Moravia, datée de la période du Développement régional, la fouille ne nous a pas permis de trouver ce type de céramique en situation. Au contraire, le rare matériel apparaissant essentiellement dans la couche 3, mais également un peu dans la couche 4 , est inédit, et constitue ce que nous avons appelé la culture Pambay. Il s'agit d'une poterie fragile, mal cuite, très 
abîmée par l'acidité du sol, et dont l'épaisseur est généralement inférieure à $0,5 \mathrm{~cm}$. Le décor n'est pas très développé mais rappelle, comme nous le verrons plus loin, certains aspects du Formatif de la région de Baños. Ces observations montrent que les couches 3 et 4, malgré leur grande dissemblance, sont liées par le matériel culturel. Les couches inférieures $(7,8$ et 9$)$ ne conservent par contre aucun vestige anthropique.

Notre interprétation de la stratigraphie est la suivante : l'occupation formative concerne les couches $3,4,5$ et 6 . Il se pourrait que l'occupation proprement dite se situe au niveau de la couche 4. Sa relative pauvreté en matériel s'expliquerait par le nettoyage systématique des aires d'habitat, que pratiquent encore les Amérindiens actuels de la région. La plus grande abondance du matériel dans la couche 3 , qui est aussi plus foncée, pourrait correspondre à l'abandon de la maison, à la reprise de la végétation et éventuellement à une chute de cendres volcaniques.

Après l'analyse stratigraphique de la coupe le long du chemin et un sondage préliminaire, le site fut fouillé par décapage sur une grande surface. Néanmoins, la profondeur de 60-100 cm du niveau anthropique et la dureté du sédiment obligèrent l'usage d'une pelleteuse, technique encore peu employée en Équateur (Figure 8, en haut, page suivante). Le décapage manuel qui s'ensuivit (Figure 8, en bas) révéla les traces et les vestiges des deux tiers d'une grande maison amérindienne, le reste ayant été détruit par les terrassements modernes et par un pillage archéologique réalisé par des huaqueros (pilleurs) il y a une vingtaine d'années.

Une fois la superficie décapée, près de vingt trous de poteau se détachèrent nettement du sol alentour, jaunâtre, par leur remplissage gris sombre et leur dessin clair. D'un diamètre moyen de $40 \mathrm{~cm}$, donc plutôt gros et correspondant aux supports principaux de l'édifice - car il y a également des piquets d'une dizaine de centimètres de diamètre -, les poteaux présentaient des profils dissymétriques très caractéristiques : de forme tronconique avec une base en pointe ou convexe, mais avec un côté généralement muni d'un palier, formé lorsque le poteau fut hissé (Figure 9, pages suivantes). En effet, pour planter un poteau de grande dimension, il faut d'abord le lever jusqu'à atteindre une inclinaison de $45^{\circ}$ et reposer alors la pointe sur un gradin dans le trou, avant de finir de l'élever et de l'insérer dans sa cavité. Par ailleurs, un poteau avait une forte inclinaison, afin de servir probablement de pilier de soutènement à la charpente, comme c'est encore parfois le cas aujourd'hui (Figure 9). Le dédoublement de certains poteaux (F. 13-14, 16-17, F. 34 - F. étant l'initiale de Feature, c'està-dire une anomalie culturelle) laisse également imaginer leur remplacement ou leur consolidation lors d'épisodes de réfection de l'édifice. Cela pourrait également expliquer la présence de certains poteaux. On peut donc supposer soit une pérennité bien supérieure à celle des cases d'aujourd'hui, soit un incident ponctuel - dû par exemple à un coup de vent provenant du nord/nordest - qui aurait demandé une réparation de fortune et le doublement de certains poteaux au sud-ouest. Soulignons toutefois que dans d'autres endroits du monde, 

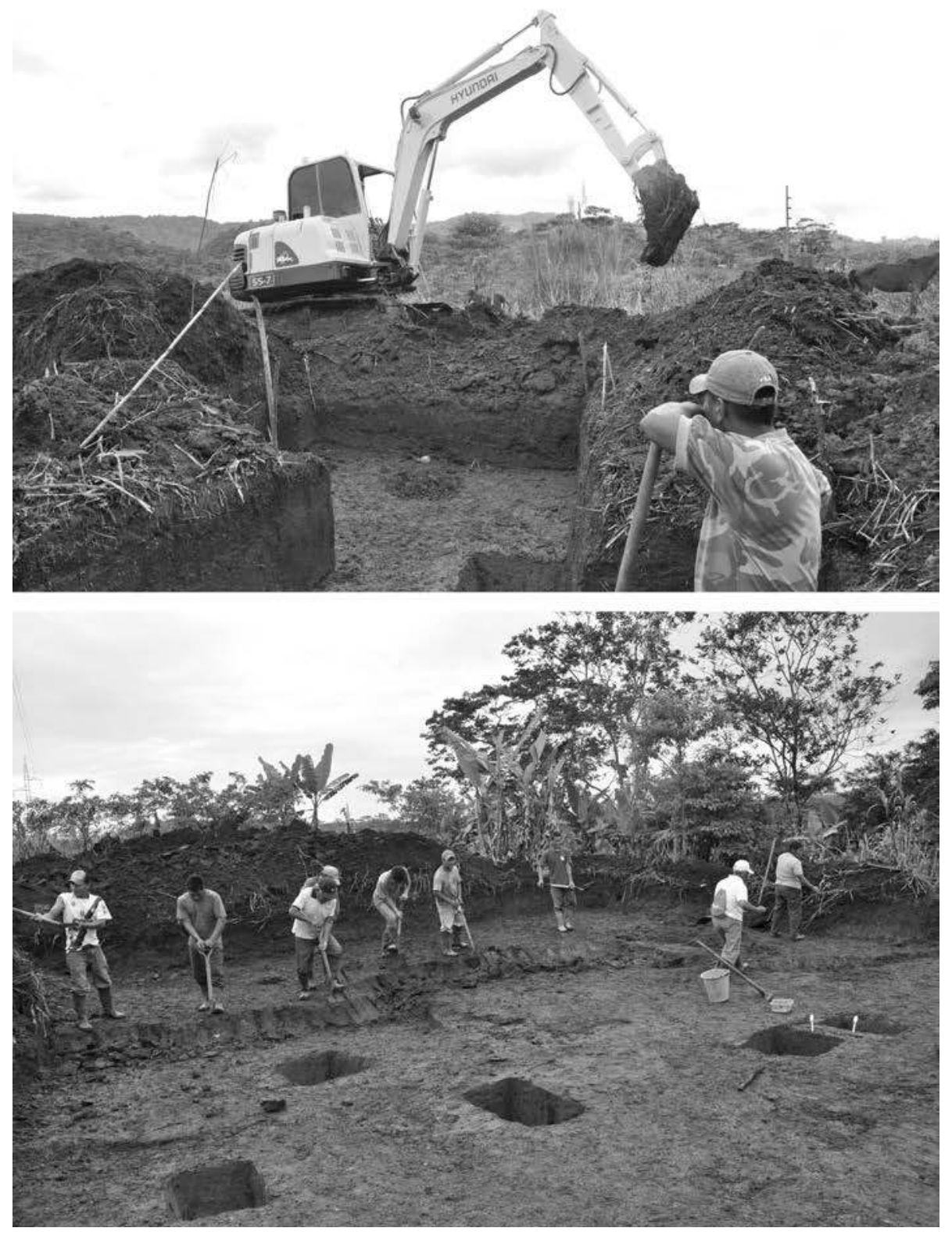

Fig. 8 - Décapage du site de Pambay à la pelleteuse, puis à la main (photographies S. Rostain). 

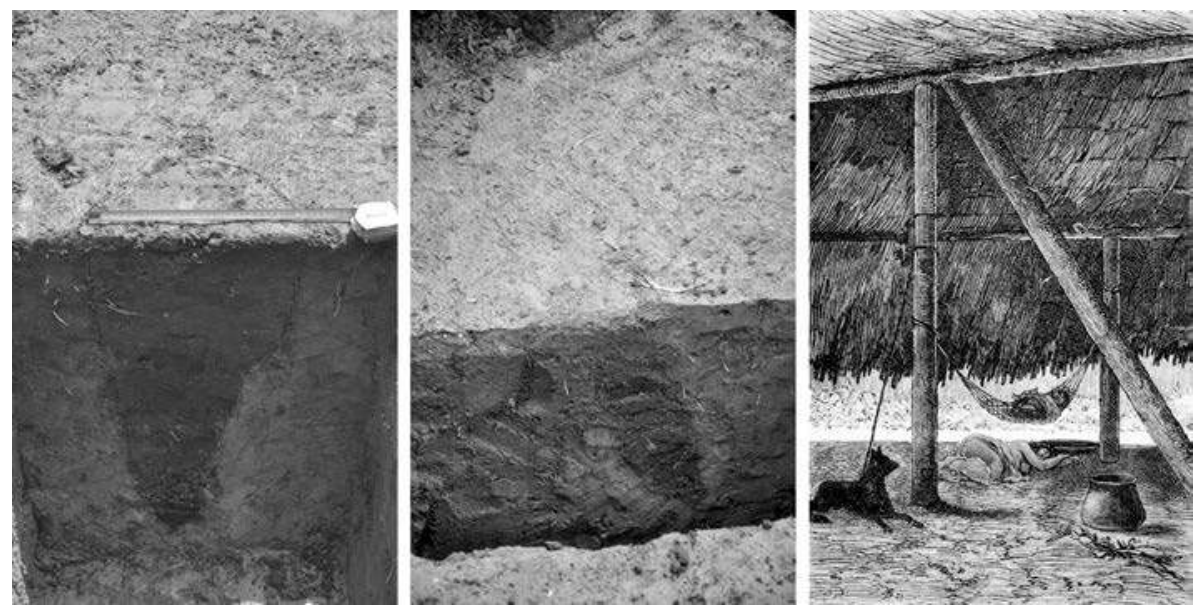

Fig. 9 - Trous de poteau de Pambay : à gauche, avec un palier sur le côté droit, vestige du hissage du poteau ; au centre, incliné ;

à droite, poteau incliné d'une « case indigène sur les bords du Samiria »,

Amazonie péruvienne (photographies S. Rostain ; dessin d'après Wiener 1882).

comme dans le site de l'Observatoire de Tokyo de la culture jomon, le remplacement de poteaux est considéré comme un indice de sédentarité, étant donné que la maison fut occupée sur une plus longue période (Imamura 1996).

La fouille par décapage, avec la vérification de chaque trou de poteau par une coupe transversale, a abouti à la découverte d'un véritable tronçon de poteau de bois, conservé de manière exceptionnelle grâce à un phénomène rare. Il a été retrouvé sous l'empreinte du trou de poteau, à quelque $3 \mathrm{~m}$ de profondeur dans l'argile anaérobie au niveau de la nappe phréatique, ce qui l'a protégé jusqu'à nos jours et a rendu cet artefact de bois unique (Figure 10, page suivante). Lors de l'extraction du poteau dont la partie immergée, ainsi que l'écorce, étaient bien conservées, les Kichwa-Canelos qui travaillaient dans la fouille ont considéré qu'il s'agissait d'un tronc de huambula, nom vernaculaire du Minquartia guianensis, l'un des bois les plus durables, les plus denses et les plus durs d'Amazonie. Connu pour être imputrescible, il est encore aujourd'hui, tout comme le palmier chonta (Bactris gasipaes), l'une des deux espèces les plus employées pour la confection des poteaux des maisons d'Amazonie équatorienne. En attendant une détermination botanique précise, on peut de toutes les façons supposer que les anciens habitants avaient choisi un bois particulièrement résistant.

La trouvaille de cet objet nous a permis d'avoir accès à certaines techniques de construction. Dans ce cas précis, au lieu de tailler un poteau, les Amérindiens ont utilisé un simple tronc ébranché en le mettant à l'envers. En effet, ils ont 


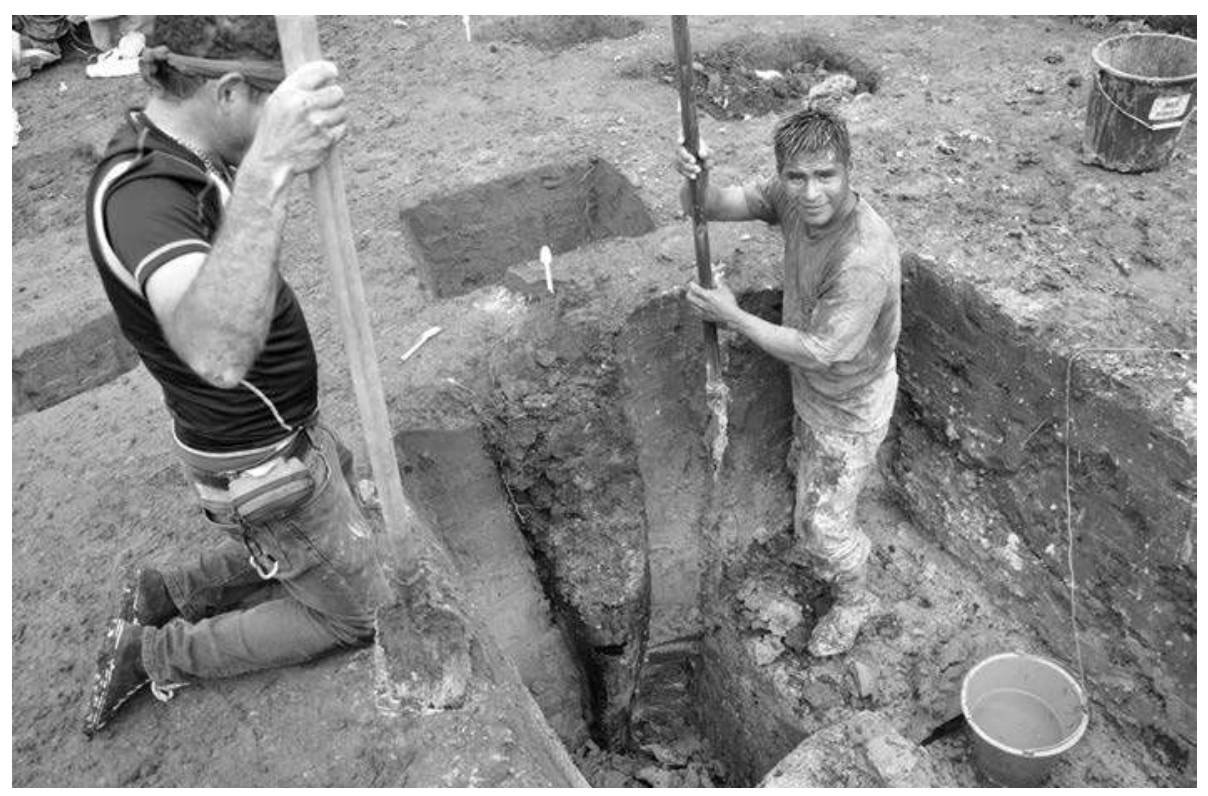

Fig. 10 - Fouille du poteau de bois de Pambay (photographie S. Rostain).

planté le tronc en fichant dans le sol l'extrémité étroite de la cime de l'arbre, tandis que la base, dense et large, se retrouvait alors en haut (Figure 11). Cette technique est astucieuse à plus d'un titre : elle limite la taille et l'élagage du tronc d'arbre, car le travail à la hache de pierre des arbres d'Amazonie souvent remplis de silice est particulièrement long et fastidieux. Elle permet ensuite, grâce à l'inertie du poteau, de l'enfoncer plus facilement dans le sol. Enfin, et c'est peut-être l'avantage le plus important, cela évite les risques de reprise de racines sur un tronc vert, conséquence fréquente en Amazonie, car la cime et le tronc n'ont pas de système d'absorption permettant de développer de nouvelles racines. Ce que les Indiens précolombiens n'avaient pas prévu, c'est que dans un terrain gorgé d'eau, le poteau allait continuer à descendre dans le sol, pour atteindre une profondeur de $3 \mathrm{~m}$ où il s'est préservé jusqu'à nos jours.

La pratique de planter un tronc à l'envers afin d'en faire un poteau n'est pas couramment documentée à travers le monde. Elle est pourtant reconnue en Alaska par exemple, tant dans le registre archéologique qu'ethnographique, notamment pour des pilotis de maison, la galette de racines ou l'empattement servant alors d'assise à la structure de la charpente (Alix 2012).

Il faut bien noter toutefois que tous les poteaux n'ont pas été confectionnés et érigés de cette manière. Quelques trous de poteau, assez larges et à fond plat ou arrondi, semblent évoquer l'utilisation de segments de tronc d'arbre massifs (40 cm de diamètre) que l'on n'aurait pas pris la peine d'épointer en 

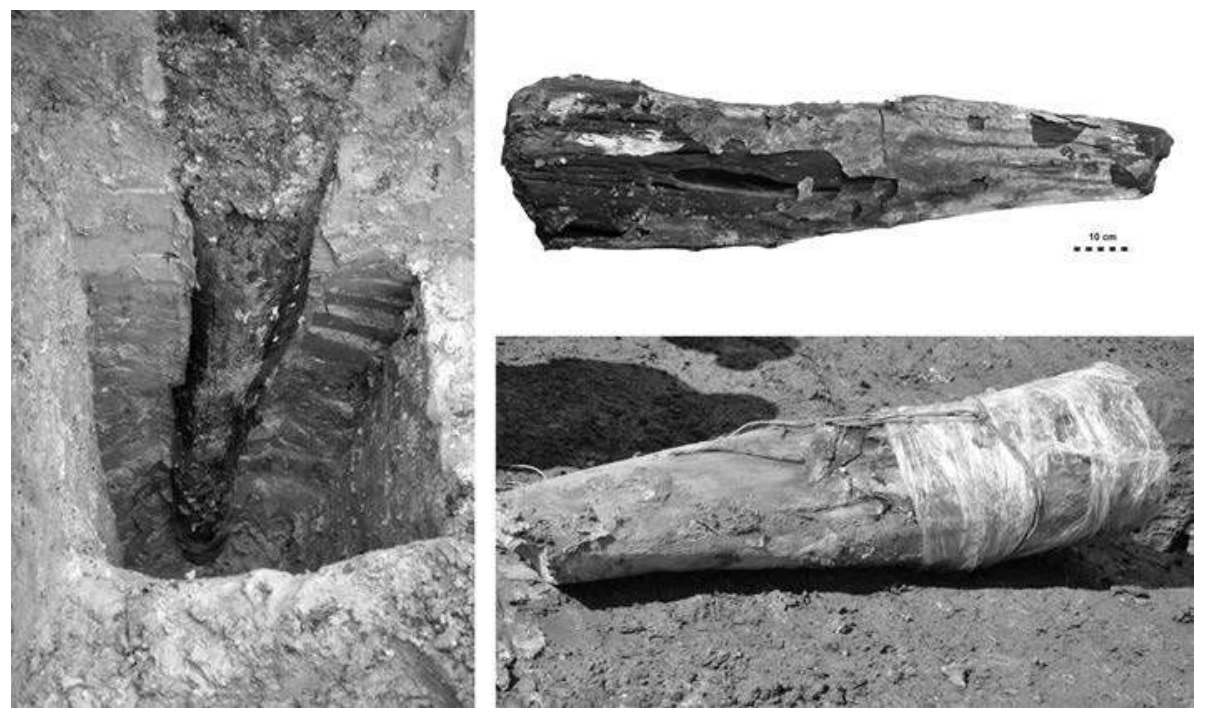

Fig. 11 - Poteau de bois de Pambay, constitué d'un tronc élagué enfoncé à l'envers dans le sol et en partie préservé dans la nappe phréatique.

Poteau juste sorti de terre en bas à droite et sec après quelques mois en haut à droite (photographie S. Rostain).

raison de leur taille (F. 11, 12, 27, 28). La disposition des trous de poteau, épais et profonds, indique une structure de forme elliptique avec des dimensions d'environ 19 m par $11 \mathrm{~m}$, longitudinalement orientée nord-nord-ouest/sud-sudest. Il s'agit là d'un modèle d'habitat très comparable à celui des populations amérindiennes actuelles de la région (Figure 11).

La grande différence entre les demeures d'hier et d'aujourd'hui réside dans le foyer. $\mathrm{Si}$, de nos jours, les Amérindiens placent simplement en étoile trois gros troncs qu'ils rapprochent du feu au fur et à mesure de leur combustion (Figure 12, page suivante), les communautés anciennes avaient construit un foyer plus élaboré. De forme circulaire et en cuvette, avec $170 \mathrm{~cm}$ de diamètre et $30 \mathrm{~cm}$ de profondeur, les parois et le fond sont tapissés de pierres, l'intérieur étant complètement rempli de charbon de bois, indiquant une intense et longue utilisation. La datation de la fin du Formatif correspond à une période durant laquelle les sociétés agro-céramiques s'organisent et se consolident dans les hautes terres andines comme sur la côte du Pacifique et, visiblement, en Amazonie.

Il faut préciser que ce foyer était localisé à moins d'un mètre d'un poteau, comme cela arrive encore parfois aujourd'hui. Toutefois, non seulement ce foyer est mieux construit que ceux actuels placés directement sur le sol, mais il était aussi le seul dans les deux-tiers fouillés de la maison. Cela constitue une différence importante avec l'habitat moderne des Shuar, Achuar et Kichwa-Canelos, 

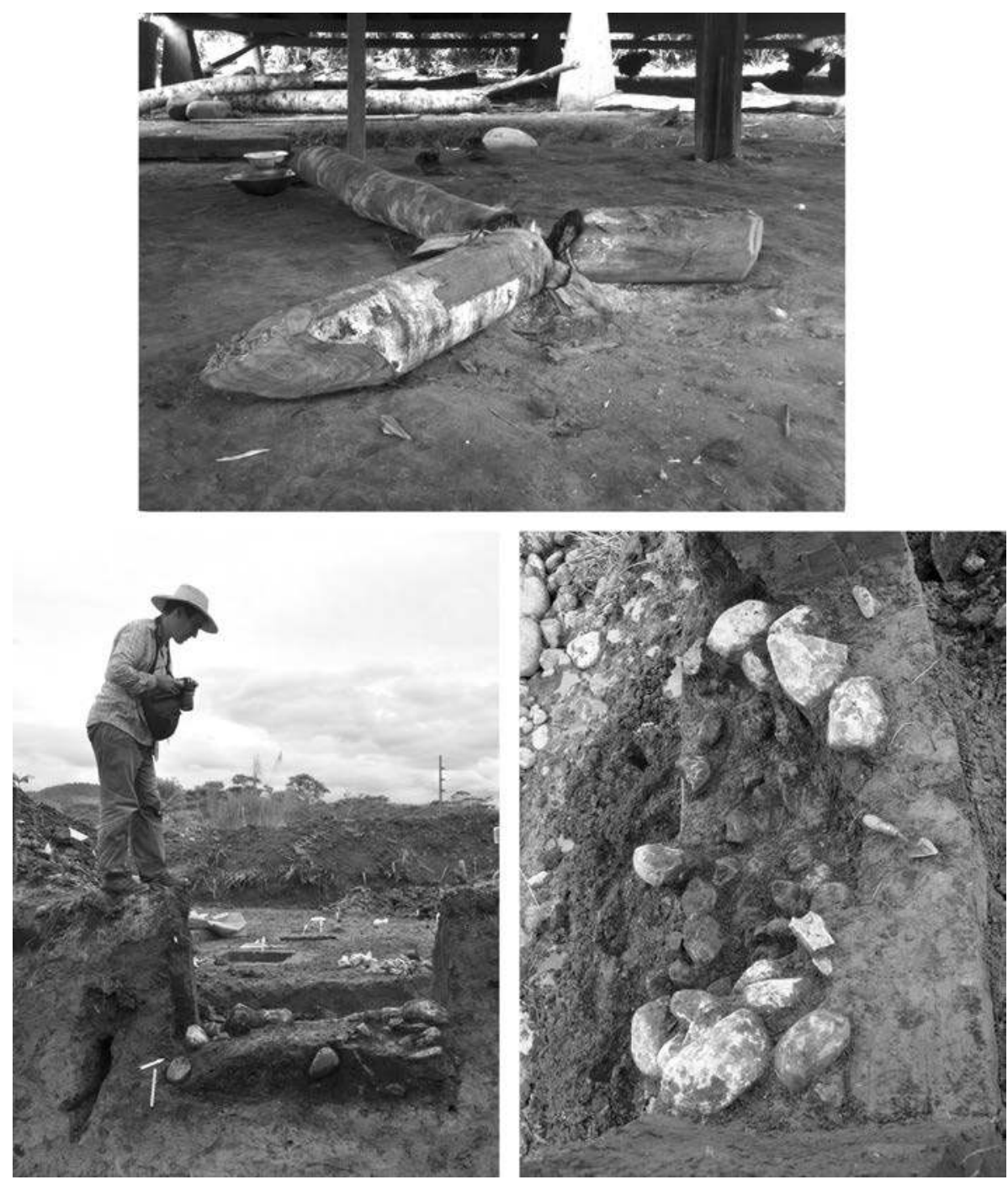

Fig. 12 - Foyers : en haut, foyer amérindien actuel du Pastaza avec trois troncs convergents ; en bas, foyer pavé de pierres à Pambay (photographies S. Rostain).

dans lequel il est courant de rencontrer plusieurs foyers sous un même toit, chaque épouse en maintenant au moins un à usage culinaire, et d'autres étant associés aux lits pour se réchauffer la nuit.

Le grand foyer de Pambay, creux et construit de pierres, n'est pas sans rappeler celui du site cérémoniel plus ancien de Santa-Ana/La Florida, situé dans une haute vallée tropicale des Andes équatoriennes. En outre, à Pambay, un 
décapage antérieur réalisé par des machines au sud de la fouille avait révélé une structure de combustion empierrée apparemment plate (Figure 13, en haut), très comparable à celles du site formatif de La Vega (Figure 13, en bas), près de Loja, dans les Andes (Guffroy 2004).

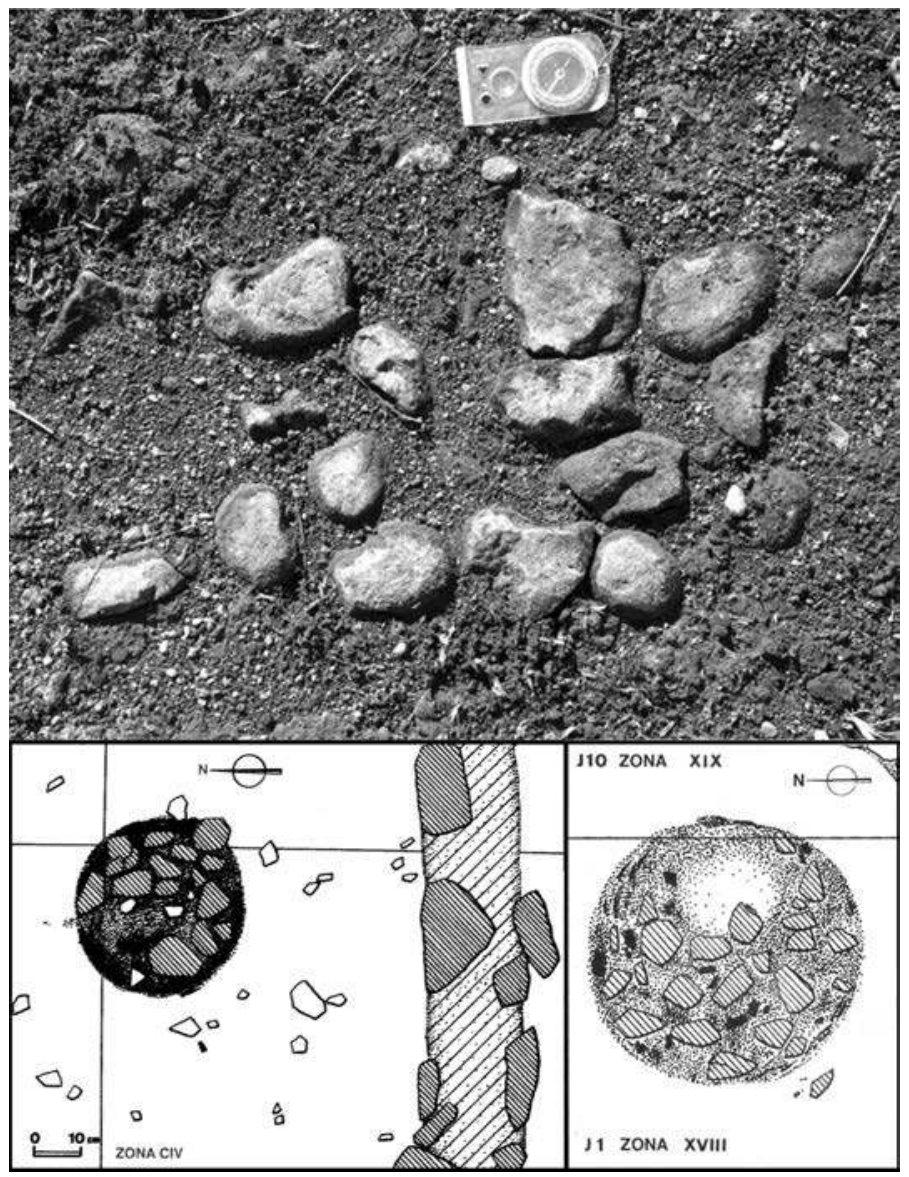

Fig. 13 - Foyers empierrés de Pambay, en haut, et de La Vega, dans les Andes, en bas (photographie S. Rostain ; dessins d'après Guffroy 2004).

Il faut enfin signaler la présence de deux larges fosses à l'extrémité nord de la maison. De forme elliptique avec des fonds légèrement dissymétriques mais néanmoins arrondis, elles font respectivement 45 et $55 \mathrm{~cm}$ de profondeur pour 85 et $70 \mathrm{~cm}$ de diamètre. Elles étaient remplies d'un sédiment noir hétérogène composé de terre, de charbon, de tessons et de quelques graviers. Elles sont similaires aux fosses de stockage découvertes dans la maison de culture Huapula (800-1200 apr. J.-C.) du site de Sangay, dont l'une était même fermée par le col 
d'une urne (Rostain 2011). Si l'on étudie l'ethnographie de la haute Amazonie, la mention de fosses dans le sol des maisons est courante. Ainsi, les Tukano d'Amazonie colombienne creusent derrière la maison commune une ou plusieurs fosses de 60 à $120 \mathrm{~cm}$ de profondeur et de 45 à $60 \mathrm{~cm}$ de diamètre (Hugh-Jones et Hugh-Jones 1996). Après avoir tapissé la cavité de larges feuilles, ils la remplissent presque entièrement d'amidon cru qui, jour après jour, est récupéré dans un récipient placé sous le tamis où des préparations alimentaires sont lavées. Il s'agit le plus souvent de manioc (Manihot sp.), mais le procédé sert également pour des graines râpées, ou des fruits de palmier. Quand la fosse est pleine, on la recouvre de feuilles puis on scelle le tout d'une couche de terre. On prendra soin toutefois, de régulièrement changer et nettoyer l'emballage de feuilles afin d'éviter l'attaque des asticots. L'amidon ainsi récupéré peut se conserver deux ans. Des pratiques similaires sont tout à fait envisageables dans le cas de Pambay.

L'analyse planimétrique des anomalies (trous de poteau, fosse et foyer) autorise plusieurs reconstitutions hypothétiques du plan de la maison. Des différentes options possibles, celle d'un édifice elliptique de plus de $200 \mathrm{~m}^{2}$ apparaît la plus plausible (Figure 14).

\section{Un mobilier archéologique épars}

Très peu de vestiges mobiliers ont été retrouvés dans la maison. En outre, ils sont peu diversifiés et se limitent à la céramique et à quelques objets lithiques. Ces derniers, très rares, ne présentent pas de traces claires d'utilisation. Il s'agit essentiellement d'une quinzaine de petits galets de dimension modeste $(4-5 \mathrm{~cm}$ de diamètre), dispersés au pied des poteaux F. 13 et 14 dans une zone de $2 \mathrm{~m}$ sur $1 \mathrm{~m}$. Il est possible que ces galets aient été des poids de filets de pêche, tels qu'ils sont encore fabriqués dans la région et, plus particulièrement, d'un épervier (appelé localement ataraya, littéralement « attrape-raie »), un filet qu'on lance en l'air et que l'on laisse couler. La corde du filet, généralement en fibre de palmier, est nouée autour de chaque poids, et encollée avec une résine végétale.

La céramique est un peu moins rare, mais plutôt mal conservée : tout au plus 200 tessons très fragmentés. À la différence des sols d'occupation de sites voisins fouillés durant le projet «Zulay »-comme Colline Moravia où le matériel céramique a été retrouvé éparpillé par petits bouts, façon puzzle -, les tessons de Pambay étaient rares et souvent isolés. Il existe toutefois une concentration à un mètre à l'ouest du poteau F. 7 (Figure 14), mais, vu son caractère exceptionnel, il est difficile de l'interpréter en termes de vestige d'aire d'activité.

Parmi les 200 tessons collectés, on distingue cinq pâtes qui correspondent également à des formes et des traitements différents. 


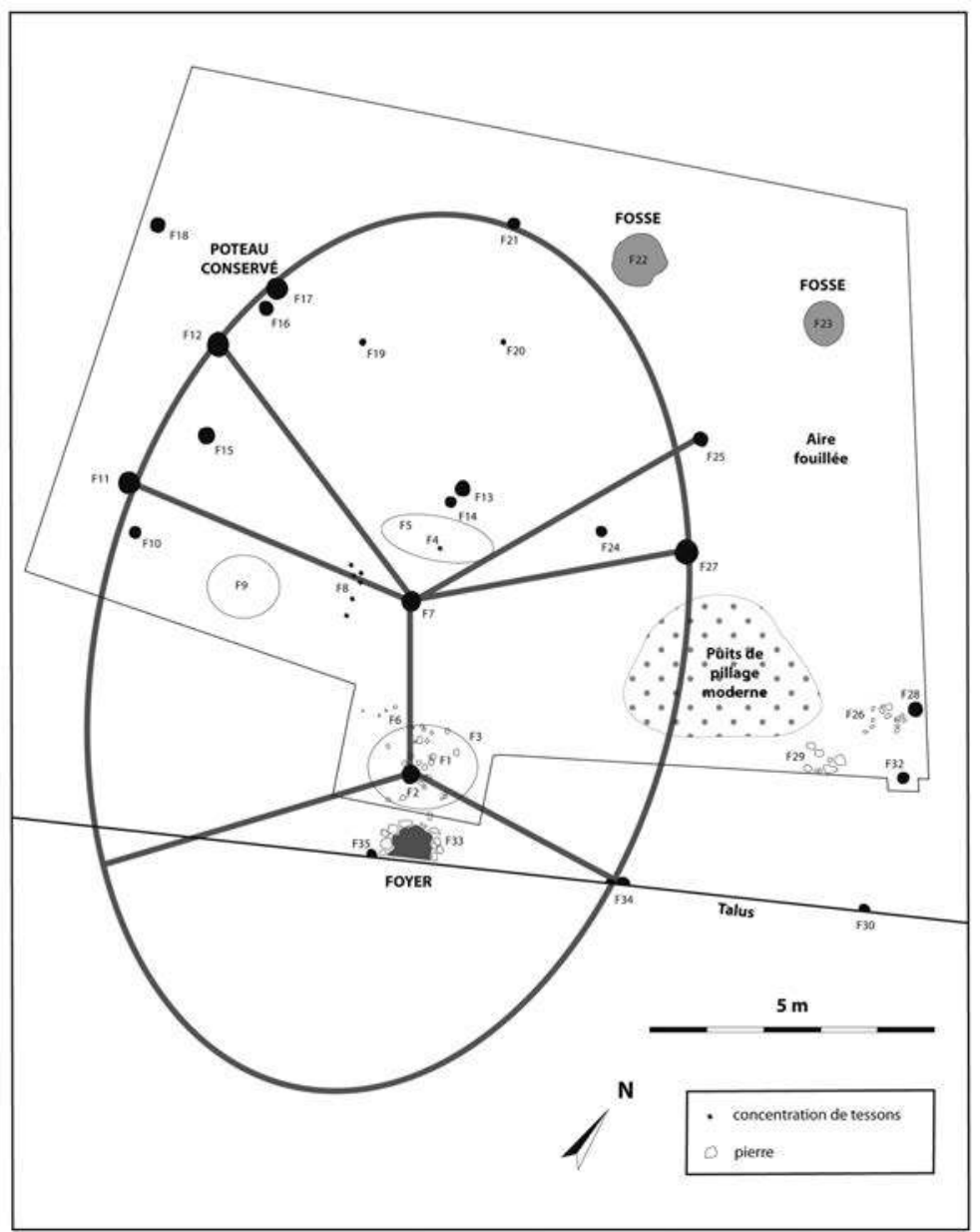

Fig. 14 - Plan hypothétique de la maison de Pambay (dessin G. de Saulieu).

La Pâte I, la plus commune avec plus de 80 tessons, est orange brique, avec un dégraissant fin constitué de particules blanches arrondies représentant environ $10 \%$ de la pâte. Elle correspond à une vaisselle à paroi peu épaisse comprise entre 0,5 et $0,2 \mathrm{~cm}$. Il s'agit surtout de récipients fermés, composés notamment des cols verticaux de jarre à bord évasé et lèvre repliée vers l'extérieur (Figure 15.2), et des bols simples évasés, à paroi convexe et lèvre repliée vers l'extérieur (Figure 15.1). La surface des tessons est soigneusement lissée.

La Pâte II est presque aussi fréquente puisqu'elle rassemble une soixantaine de fragments. C'est une pâte beige, rappelant la Pâte I, mais avec un dégraissant plus abondant $(30 \%)$. Elle est également hétérogène et moins compacte que la Pâte I, et présente des surfaces moins bien lissées. Les récipients y sont très 

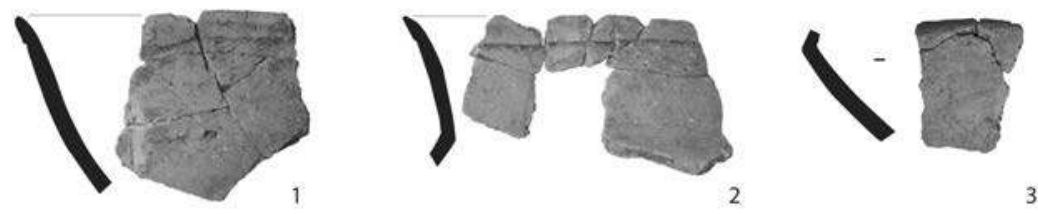

3
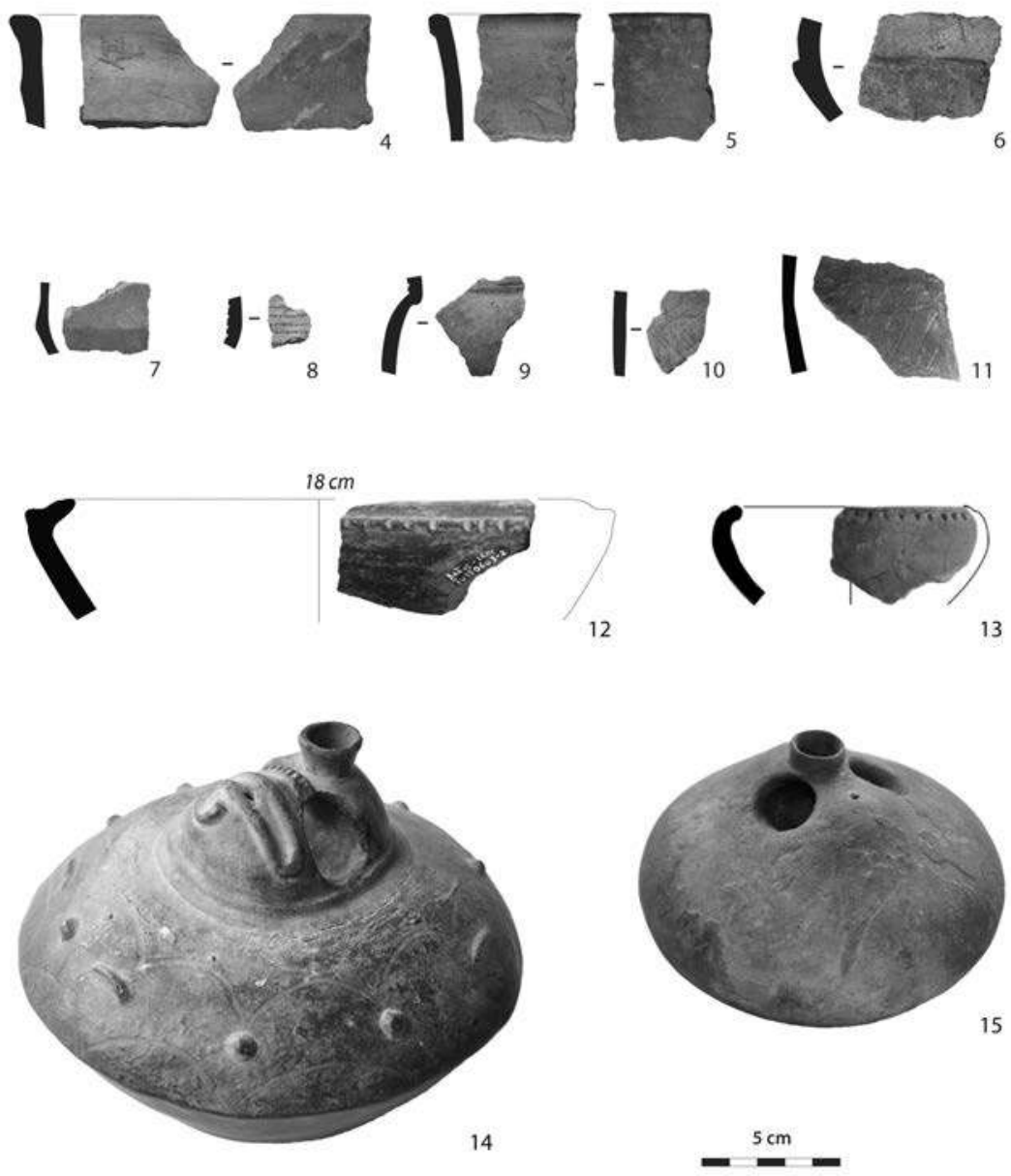

Fig. 15 - Céramiques formatives du bassin du Pastaza. 1 à 10 : complexe Pambay, site de Pambay. 11 et 12 : Formatif de la région du Tungurahua, haut Pastaza. 13 : récipient Pambay, site de Colline Moravia. 14 et 15 : bouteilles à anse en pont asymétrique, provenant de la province de MoronaSantiago qui, bien que non datées, sont probablement du Formatif tardif (photographies G. de Saulieu, S. Rostain et C. Jaimes Betancourt). 
souvent à paroi fine (entre 0,6 et $0,8 \mathrm{~cm}$ d'épaisseur), et possèdent fréquemment une carène (Figure 15.3).

La Pâte III, avec ses 30 fragments, possède une couleur brune à grise. La pâte est très typée avec un dégraissant abondant (30\%) de grains blancs de moins de $0,1 \mathrm{~cm}$ de diamètre. Si l'extérieur est souvent lissé avec soin, l'intérieur des récipients, la plupart du temps des écuelles carénées à bord vertical, présente une surface intérieure brunie et polie noir brillant (Figures 15.4 et 15.5). L'épaisseur des tessons va de $0,2 \mathrm{~cm}$ à $0,6 \mathrm{~cm}$.

Les 30 tessons de Pâte IV ont une texture grossière : le dégraissant abondant (30 à $40 \%$ ) peut comporter des grains de plus de $3 \mathrm{~mm}$. La surface des récipients laisse généralement entrevoir un lissage grossier pour des récipients carénés de type écuelle et jarre, avec des parois de 0,6 à 0,8 cm d'épaisseur (Figure 15.6).

On notera pour finir un tesson fin et caréné, récupéré à proximité du foyer, qui pourrait représenter une dernière Pâte V. Elle est de couleur crème clair avec un dégraissant très abondant $(50 \%)$ constitué de grains blancs de moins de $1 \mathrm{~mm}$ (Figure 15.7).

Les décors sont peu nombreux. Des lignes parallèles diagonales réalisées au traçage sont présentes sur deux tessons (Pâtes I et IV, Figure 15.6), alors qu'un seul tesson présente de l'engobe rouge vif (Pâte V, Figure 15.7). L'intérieur noir brillant est réservé aux récipients ouverts de Pâte III (Figures 15.4 et 15.5). Notons pour finir que deux tessons de Pâte I sont décorés de lignes incisées et profondément ponctuées (au peigne ?) (Figures 15.8 et 15.9).

Malgré son état fragmentaire, ce matériel montre deux choses. On ne peut d'abord qu'être étonné de sa diversité malgré la taille limitée de l'échantillon. Ensuite, son insertion dans le paysage culturel contemporain de la fin du Formatif équatorien semble évident (Rostain et Saulieu 2013). Il faut remarquer que la carène, très fréquente dans le matériel de Pambay, est un élément omniprésent dans les différentes cultures céramiques de la fin de cette époque, que ce soit Machalilla et Chorrera sur la côte pacifique (Meggers et al. 1965) ou Cotocollao dans la cordillère (Villalba 1988) et les cultures apparentées comme celle de Loma Pucara (Arellano 1997). Il est à noter que les cultures céramiques d'Amazonie péruvienne qui sont contemporaines de Pambay, à savoir Tutishcainyo Tardif et Cobichaniqui (Lathrap 1970), affichent également une très forte popularité de formes carénées et, notamment, des formes ouvertes.

Quant aux sites formatifs des versants du volcan Tungurahua (Le Pennec et al. 2013), à environ $40 \mathrm{~km}$ à l'ouest, ils présentent aussi un matériel caréné bien daté. Ces sites ont été identifiés par le volcanologue Jean-Luc Le Pennec à l'occasion d'un programme de recherche de plusieurs années sur l'histoire du volcan Tungurahua, dominant les sources du Pastaza. Les résultats montrent que des habitats précolombiens ont été détruits par une éruption majeure vers 1100 av. J.-C. (calibré). L'échantillon céramique, bien que très faible (38 tessons) et provenant de trois localités, comporte quelques traits 
diagnostiques qui autorisent des comparaisons avec le matériel contemporain du complexe Pambay. On trouve notamment des fragments de bol, dont la carène, très proche de l'ouverture, est décorée d'encoches verticales (Figure 15.12). Si cette forme est très semblable à des récipients de la culture contemporaine de Cotocollao dans la région de Quito, elle fait également beaucoup penser à des tessons découverts dans les contextes archéologiques Pambay, fouillés sur le site de Colline Moravia (Figure 15.13). L'on pourra aussi mentionner une technique décorative commune, consistant en lignes diagonales parallèles exécutées par traçage superficiel (Figures 15.10 à Pambay et 15.11 pour le site formatif dans le matériel du Tungurahua). En fin de compte, force est de constater que les décorations sont peu développées dans l'un et l'autre des complexes céramiques qui partagent avant tout un certain goût pour la sobriété.

La céramique avec l'intérieur noir brillant que l'on a découverte sur le site de Pambay (Figures 15.4 et 15.5) précède quant à elle des développements amazoniens ultérieurs bien connus dans la vallée de l'Upano située à environ $100 \mathrm{~km}$ au sud de là. En effet, c'est avec la culture upano entre 500 av. J.-C. et 400 apr. J.-C. que l'on voit l'essor d'un décor fondé sur des motifs géométriques réalisés en bandes rouges entre incisions (Rostain 2010). L'une des formes les plus populaires est le bol, ou l'écuelle carénée, à bord vertical, dont l'intérieur noir brillant est obtenu par brunissage et polissage, ce qui est exactement le cas des tessons de Pambay, pourtant plus anciens.

Vue la chronologie du matériel, il n'est pas interdit de penser que le complexe Pambay fait partie du «Flash horizon » que Warren DeBoer (2003) propose vers 800 av. J.-C., et peut-être même un peu avant, pour la haute Amazonie. À la grande popularité des récipients ouverts à carène, il faut en effet ajouter pour cette vaste région partagée entre le Pérou et l'Équateur, la présence récurrente de bouteilles à anse en pont asymétrique (Figures 15.14 et 15.15), dont l'usage reste encore énigmatique. Ce qui est certain, c'est que les bouteilles entières, ou leurs fragments, apparaissent en nombre dans les provinces de Morona-Santiago et Pastaza, y compris à proximité de Puyo (Duche Hidalgo et Saulieu 2009), mais très rarement en situation primaire. La pâte, compacte et homogène, ainsi qu'une cuisson souvent réalisée en atmosphère réductrice, fait penser à la Pâte III de Pambay, celle-là même qui a la particularité d'un décor noir brillant dans les bols et écuelles à bords verticaux.

En dépit de son échantillon restreint, la céramique de Pambay manifeste une insertion évidente dans le contexte chronologique et culturel très dynamique du Formatif tardif équatorien et amazonien. La tradition céramique de Pambay ne dépare pas des cultures contemporaines du versant oriental et des Hautes Terres andines, ni des grandes cultures côtières, ce qui atteste de relations lointaines. En outre, elle semble annoncer des styles céramiques amazoniens qui s'affirmeront quelques siècles plus tard et témoigne ainsi de l'existence de processus culturels en cours en Amazonie, dont nous n'avons encore qu'une très vague idée. 
On mentionnera pour terminer que le matériel céramique et lithique récolté lors du projet sur le site de Colline Moravia, contemporain et voisin de quelques kilomètres de Pambay, a autorisé une analyse archéobotanique de grains d'amidon qui a mis en évidence la présence de diverses plantes comme le maïs (Zea mays), le manioc (Manihot esculenta), le haricot (Phaseolus sp.) et d'autres légumineuses (Fabaceae), mais également le cacao (Theobroma sp.), consommé très anciennement en Amazonie équatorienne (Pagán Jiménez et Rostain 2014). Mais ce sont peut-être les liens établis avec les hautes terres qui retiennent le plus l'attention grâce à la reconnaissance de l'ulluque (Ullucus tuberosus), tubercule comestible généralement cultivé dans les Andes entre 2600 et $3800 \mathrm{~m}$ audessus du niveau de la mer. Pour l'instant, nous ne savons pas à quel point cette plante peut fructifier ou s'adapter à l'altitude de Colline Moravia, entre 1100 et $1200 \mathrm{msnm}$, soit $250 \mathrm{~m}$ plus haut que Pambay. Il faut toutefois noter que la région est très fraîche en raison d'une insolation moins importante qu'ailleurs en Amazonie. En effet, la cordillère forme une barrière où s'accumulent les nuages, les brumes et l'humidité qui mitigent les pics de températures. Puyo, nom de la ville à proximité de laquelle se trouve Pambay, signifie d'ailleurs en quichua « brouillard». Toutefois, nous sommes plutôt enclins à interpréter cette découverte de l'ulluque à Colline Moravia comme le résultat d'échanges de biens, d'idées et de croyances entre les cultures des Hautes et Basses Terres.

Quoi qu'il en soit, l'ensemble des données archéobotaniques comparées avec celles obtenues dans d'autres sites formatifs comme Sangay sur le piémont amazonien des Andes et Santa-Ana/La Florida sur les versants orientaux des Andes (Rostain 2011 ; Zarillo 2012), laisse supposer que la diète végétale reposait à cette époque principalement sur le maïs (Zea mays), le manioc (Manihot esculenta) et les haricots (Phaseolus sp.), et que le cacao (Theobroma sp.) était également consommé, peut-être de préférence durant des rituels, ainsi que la coca (Erythroxylum coca). En revanche, on remarquera l'absence totale d'outils de mouture à Pambay, car ni meule, ni molette n'ont été retrouvées. On peut en déduire que, soit la maison a été méthodiquement nettoyée lors de l'abandon, soit certaines activités étaient réalisées ailleurs. À ce propos, notons qu'apparemment un autre édifice était construit à l'est, comme le suggère la présence de trois trous de poteaux alignés (F. 28, 32, 30).

\section{Comprendre l'habitat formatif d'Amazonie équatorienne}

La découverte essentielle du projet «Zulay », qui englobe tous les aspects de la fouille, est pourtant bien celle d'une maison formative. Si l'occupation formative a été clairement mise en évidence dans le site plus occidental de Colline Moravia, il n'a pourtant pas été possible de retrouver le plan de l'habitat comme à Pambay. 
Les maisons d'époque formative connues en Équateur se trouvent toutes sur la côte pacifique ou dans les Hautes Terres andines, aucune n'étant signalée en Amazonie. Un coup d'œil rapide permet de voir que la maison de Pambay, spacieuse et elliptique, ne partage que peu de points communs avec l'habitat formatif contemporain des Andes, que ce soit celui de La Vega à Catamayo (Loja), sorte de case en auvent construite sur une base pierreuse en arc-de-cercle (Guffroy 2004), ou celui de Cotocollao avec ses constructions rectangulaires (Villalba 1988).

Un rapprochement plus intéressant semble pouvoir être fait avec les maisons Valdivia de la côte pacifique, car elles y sont toujours elliptiques (Zeidler 1984; Guillaume-Gentil 2013). Toutefois, ces dernières sont beaucoup plus courtes que la maison de Pambay, puisque la longueur de la structure 1 de Real Alto oscille entre 10 à $12 \mathrm{~m}$ (Zeidler 1984) et n'atteint que 3,10 m à Loma Alta (Damp 1984), ce qui semble expliquer pourquoi les poteaux intérieurs sont peu nombreux, contrairement à Pambay. Certaines d'entre elles possèdent des murs en torchis. Jonathan Damp (1984) fait remarquer que les activités domestiques (feu, taille de la pierre, filage du coton) prennent place en dehors de la maison, qui ne sert finalement que comme chambre à coucher. Comme à Pambay, nous voyons ici la présence de fosses extérieures qui pourraient être à l'origine des structures de stockage. De même, à Cotocollao, on remarque également la présence de fosses de stockage, notamment à l'intérieur des maisons (Villalba 1988 ; Zeidler 2008). En revanche, le foyer principal de la structure 1 de Real Alto est excentré et se trouve dans la moitié sud de la maison, après la cloison qui garde l'entrée (Zeidler 1984). On notera que cette maison possède la même orientation nord-nord-ouest/sud-sud-est que celle de Pambay. Toute une répartition des activités a pu être proposée à l'intérieur de la maison : un espace de couchage, deux zones de préparation des coquillages, des aires de circulation, des surfaces brûlées correspondant sans doute à des foyers secondaires (chauffage nocturne des lits ?), etc. Malheureusement, le sol de la maison de Pambay a été, semble-t-il, méticuleusement nettoyé avant l'abandon, comme nous l'avons évoqué. Il est donc impossible de comparer les aires d'activité.

Encore plus que les maisons Valdivia, l'habitat de Pambay présente des caractéristiques très proches de celles de cases actuelles des Achuar, des Shuar et des Kichwa de la région (Figure 16), tant dans le plan que dans les dimensions, tout comme de celles de leurs ancêtres de culture huapula (Rostain 2011). Cette similitude plaide pour une pérennité technique remarquable à travers le temps. Les différences sont de deux ordres. Pour ce que nous pouvons en juger, les techniques anciennes, inféodées à un outillage de pierre, sont beaucoup plus pragmatiques qu'actuellement. En effet, la diversité des types de poteaux de la maison laisse imaginer une volonté d'économie de travail. L'utilisation actuelle d'outils en métal, notamment de la machette, permet une sélection et une standardisation facile et plus rapide des matériaux de construction. C'est peut-être aussi dans ce sens qu'il faut interpréter les dédoublements de poteaux (F. 13-14, 
16-17, 34) correspondant à des épisodes de réparation. La seconde différence majeure se trouve dans le foyer, car si les foyers modernes sont constitués de trois troncs radiaux que l'on avance progressivement vers le feu, celui de Pambay est nettement plus élaboré, avec la construction d'une large et profonde cuvette en pierres (Figure 12). La présence d'une série de trous de poteau à l'est et des restes d'un second foyer à $15 \mathrm{~m}$ au sud du premier, laisse supposer l'existence d'un autre édifice, peut-être lié au premier, mais le terrain, complétement râpé par des travaux modernes à cet endroit, ainsi que le manque de temps empêchaient de pousser plus avant nos recherches. En revanche, le second foyer empierré du sud permet de conjecturer qu'une autre cabane, au moins, accompagnait la première.

Par ailleurs, on peut se rappeler que des foyers présentant des caractéristiques similaires dans le diamètre, la concavité et la localisation au pied d'un poteau, existent dans les malocas actuelles des Miraña et Uitoto du Caquetá, en Colombie. Formés par la consumation des déchets après la construction de l'édifice, ces foyers, appelés mambiaderos en espagnol, sont intiment liés à l'usage de la coca car on y brûle les feuilles dont les cendres seront incluses dans la poudre de coca destinée à être mâchée. En outre, ce foyer, localisé près d'un poteau de soutènement, est le lieu d'autorité du maître de maisonnée assis sur son banc où il reçoit ses visiteurs et partage la coca avec eux. C'est un espace qui permet au maître des lieux d'affirmer « Je suis chez moi ! (Karadimas, com. pers. 2015).

De toutes les façons, la similitude avec l'habitat indigène amazonien moderne ne laisse pas de surprendre. Le plan elliptique, l'orientation générale de la maison, nord-est/sud-ouest, comme sa position au sommet d'une petite colline entre deux ruisseaux, coïncident curieusement avec la préférence traditionnelle des Amérindiens actuels. Il est toutefois nécessaire de préciser notre pensée : cette similitude n'est peut-être que formelle et ne signifie pas forcément que les modes de vie et les structures sociales étaient identiques.

Au-delà de son ancienneté extraordinaire pour la région, cette demeure est rare dans l'archéologie d'Amazonie, car très peu de maisons y ont été trouvées. Le long du même piémont amazonien d'Équateur, plus au sud, on peut citer l'habitat de culture huapula, daté entre 800 et 1200 apr. J.-C., dans le site de Sangay de la vallée de l'Upano, où l'étude spatiale de la dispersion des traces et vestiges sur un sol d'occupation a permis la restitution d'une demeure amérindienne (Rostain 2006, 2011).

\section{Conclusion}

Sorti d'une très longue hibernation, le Formatif amazonien d'Équateur se dévoile peu à peu depuis une quinzaine d'années. Ce furent en premier lieu les spectaculaires complexes de monticules artificiels de terre de la vallée de l'Upano qui révélèrent de multiples facettes de sociétés denses de terrassiers et commerçants du piémont oriental des Andes, ainsi que de leurs prédécesseurs 

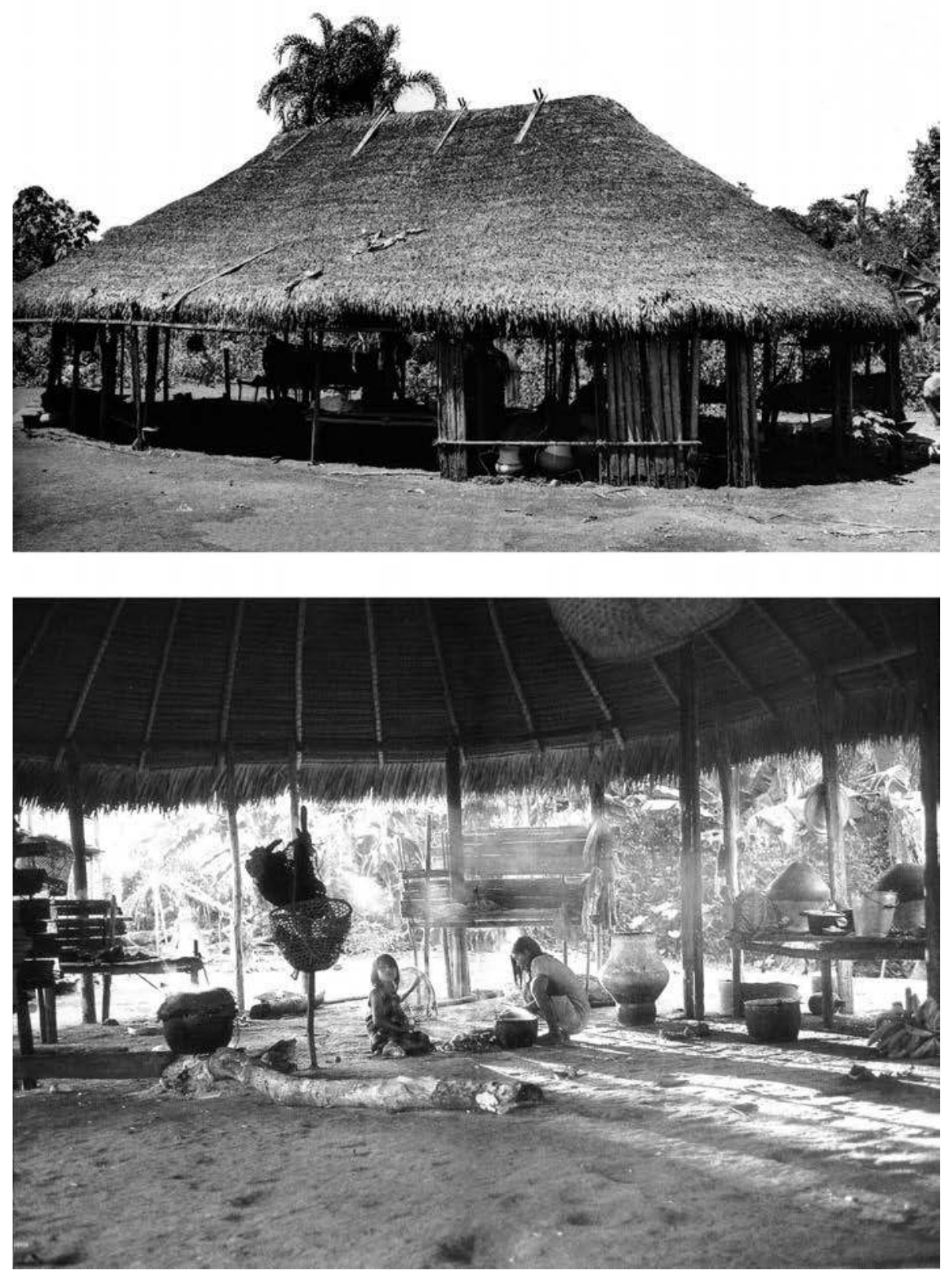

Fig. 16 - Maison achuar moderne du moyen Pastaza (photographies P. Descola). 
(Rostain 1999). Ces communautés, localisées à la frontière des Hautes Terres et des Basses Terres, diffusèrent en effet leur céramique, au décor très spécifique de dessins géométriques peints en rouge et délimités par des incisions, jusque loin dans les Andes. Cette brillante culture connut une fin tragique, comparable à celle des habitants de Pompéi, puisqu'ils subirent une effroyable éruption du proche Sangay qui noya tout le nord de la vallée de l'Upano sous une épaisse couche de cendres volcaniques. Un des aspects les plus surprenants de la recherche, menée à la fin des années 1990, fut la densité extraordinaire de tertres et de chemins creusés qui couvraient l'ensemble de la vallée de l'Upano, sur des dizaines de kilomètres. En fait, c'est quasiment toute la superficie des hautes terrasses de la rivière qui avait été modifiée par l'homme il y a quelque 2500 ans.

Enfin, dernière en date, la découverte de la maison antique de Pambay est une nouvelle " broche de oro » sur la révélation progressive du plus lointain passé humain de l'Amazonie équatorienne. Elle a été permise à la faveur de la réalisation d'un programme de recherche interdisciplinaire dans la région de Puyo, mais également grâce à la mise en place de fouilles horizontales par décapage de grande surface. Bien qu'encore peu populaire en Amazonie, et moins encore en Équateur, c'est pourtant la meilleure méthodologie pour la mise en évidence des restes d'un habitat et la compréhension synchronique des diverses activités qui y furent pratiquées. Il faut en effet se rappeler qu'il n'est pas si loin le temps où l'on fouillait en Amazonie exclusivement par « cabine téléphonique » dans l'espoir que ces sondages d'un mètre carré révèlent une stratigraphie, pourtant peu probable dans ce milieu. Cette méthode avait notamment été popularisée par Betty J. Meggers, alors chef de file de l'archéologie amazonienne, qui recommandait en outre d'établir la chronologie sur la base d'une stratigraphie artificielle censée pallier l'homogénéité du sédiment et restituer la succession des événements passés. Moins communs aujourd'hui, les sondages stratigraphiques sont souvent remplacés par des tranchées, dont la conception n'en est pourtant pas si éloignée.

Une autre contrainte contribue à expliquer la rareté d'habitats précolombiens reconnus en Amazonie. En effet, si des forêts de trous de poteau ont été mises au jour dans différentes fouilles amazoniennes, les reconstitutions de l'habitat originel ne sont pas légions. L'absence de stratigraphie et la multiplicité de traces sur un même niveau empêchent souvent une bonne lecture et interprétation des anomalies, laissant au plus deviner une superposition de multiples occupations en un même lieu. Ici, nous avons eu la chance de rencontrer un édifice qui a été abandonné et rapidement recouvert, probablement par des phénomènes en partie volcaniques.

Les fouilles par décapage de grandes surfaces sont exceptionnelles, et encore trop souvent menées selon des principes stratigraphiques occultant une vision horizontale de l'espace. Malgré leur évidente efficacité en milieu tropical humide, ce type d'excavation est encore peu pratiqué en Amazonie. Il est surtout populaire dans les fouilles préventives, car les aménageurs se chargent 
naturellement d'ouvrir de grandes surfaces qui peuvent être ensuite observées par les archéologues. Néanmoins, c'est alors bien le commanditaire qui décide du lieu et le circonscrit, obligeant le chercheur à suivre son choix sans possibilité de s'écarter de la ligne arrêtée. Là encore, le terrain étudié demeure restreint car limité par l'étendue concernée par les travaux. Il en résulte ainsi des plans tronqués de sites et une interprétation difficile de l'habitat à partir des innombrables trous de poteau et autres anomalies révélées. Dans le cas de fouilles programmées, les fonds alloués sont souvent bien insuffisants pour utiliser du matériel lourd comme les pelleteuses et une équipe d'ouvriers suffisamment nombreux. En outre, il est fréquent que de tels projets de recherche se réalisent dans des aires éloignées où l'accès à des machines est difficile, voire impossible.

C'est donc une opportunité exceptionnelle d'avoir pu organiser des fouilles par décapage étendu dans ce milieu amazonien, tout comme de découvrir des traces si claires d'un des premiers logis précolombiens. Si l'on s'en donne les moyens, d'autres devraient apparaître.

En conclusion, les programmes scientifiques « Sangay-Upano/Río Blanco » et «Zulay », réalisés à quinze ans d'écart dans deux vallées piémontaises voisines d'Équateur, ont chacun apporté leur lot de résultats novateurs et contribué à l'enrichissement de l'archéologie et de l'écologie historique des basses terres tropicales d'Amérique du Sud. Tout d'abord, de longues chronologies locales furent définies dans chacune des aires, commençant au Formatif récent pour s'achever de nos jours (Figure 3). En outre, la consommation de certaines plantes amazoniennes et andines a pu être déterminée avec précision dans les deux cas. De grands événements naturels avec un impact décisif sur les habitants, comme des éruptions volcaniques catastrophiques, ont été révélés à chaque fois. Ensuite, des transformations anthropiques du paysage par terrassements ont été mises en évidence. Si, dans le cas de l'Upano, des tertres ont bien été construits par les populations précolombiennes, les élévations des terrasses du Pastaza sont, elles, parfaitement naturelles et résultent d'avalanches volcaniques, bien qu'elles aient été parfois partiellement aménagées et habitées - alors qu'elles étaient jusqu'alors considérées comme entièrement édifiées par les hommes (Rostain et al. 2014). Enfin, des fonds de maisons ont été découverts et étudiés spatialement dans chaque vallée, apportant des informations précises et originales sur les peuples précolombiens du piémont oriental des Andes.

Il n'est pas excessif d'affirmer que les contributions majeures du programme « Zulay » ont été l'élaboration d'une chronologie du bassin du Pastaza et la mise au jour de la plus ancienne cabane amazonienne. Les données obtenues pour l'instant montrent l'existence d'occupations humaines d'horticulteurs sédentaires céramistes à la fin du Formatif, vers 1500-1300 av. J.-C. (calibré), c'est-à-dire contemporains de la culture machalilla et des phases terminales de la culture valdivia (Zeidler 2008) sur la côte pacifique, ainsi que des cultures 
challuabamba (Greider et al. 2009), catamayo B (Guffroy 2004) et cotocollao (Villalba 1988) dans les Andes équatoriennes.

Si l'on sait aujourd'hui que l'Amazonie fut habitée de longue date avant l'arrivée des Européens et que la densité démographique y fut bien plus élevée que ce que l'on supposait, on peut toutefois s'étonner, à la suite d'Eduardo Neves (2007), de voir que l'on bute sur des aires, ou des périodes, lacunaires. Il est étonnant de voir que le Formatif tardif du Pastaza, qui n'est probablement pas la première occupation humaine de la région, repose directement sur le substrat géologique, comme s'il succédait à une période d'intense érosion et de lessivages systématiques. Et cela en totale contradiction avec l'enfouissement postérieur au Formatif tardif de Pambay qui montre une intense accumulation probablement d'origine volcanique et rapidement stabilisée par la végétation.

En dépit de ces vidanges et trop pleins, la fouille de Pambay laisse entrevoir que la découverte du Formatif d'Amazonie ne sera pourtant pas un tonneau des Danaïdes. *

* Manuscrit reçu en mars 2015, accepté pour publication en septembre 2015.

Remerciements - Aux communautés amérindiennes du haut Pastaza et à Carlos Duche Hidalgo. Au ministère des Affaires étrangères, au CNRS, à l'Institut de recherche pour le développement, au programme ECOS-Sud de l'université Paris-13 et au musée Ethnoarchéologique de Puyo, pour avoir financé le projet interdisciplinaire et international «Zulay, le portail précolombien de l'Amazonie ». À Philippe Descola pour ses photographies.

\section{Références citées}

Alix Claire

2012, « Using wood on King Island, Alaska », Études/Inuit/Studies, 36 (1), p. 89-112. Arellano Jorge

1997, « Loma Pucara, un asentamiento del Formativo tardio en el valle de Cebadas, Sierra Central del Ecuador », Fronteras de investigacion, 1 (1), p. 78-100.

DAMP Jonathan

1984, « Architecture of the early Valdivia village », American antiquity, 49 (3), p. 573-585.

DeBoer Warren R.

2003, "Ceramic assemblage variability in the Formative of Ecuador and Peru ", in Archaeology of Formative Ecuador, J.S. Raymond et R.L. Burger (éd.), Dumbarton Oaks Research Library and Collection, Washington, p. 289-336.

Duche Hidalgo Carlos et Geoffroy de Saulieu

2009, Pastaza precolombino, datos preliminares (y catalogo del Museo etno-arqueológico de Puyo y del Pastaza), Abya-Yala, Quito.

Grieder Terence, James D. Farmer, David V. Hill, Peter W. Stahl et Douglas H. Ubelaker 2009, Art and archaeology of Challuabamba, Ecuador. An Ancient Andean society of the Peruvian north coast, University of Texas Press, Austin. 
GuFrroy Jean

2004, Catamayo precolombino. Investigaciones arqueológicas en la provincia de Loja (Ecuador), IFEA/IRD (Travaux de l'Institut français d'études andines, 164), Paris.

Guillaume-Gentil Nicolas

2013, Cinco mil años de historia al pie de los volcanes. Implantación, población y cronología en Ecuador, Flacso/Abya-Yala, Quito.

Hugh-Jones Christine et Stephen Hugh-Jones

1996, « La conservation du manioc chez les Indiens Tukano : technique et symbolique », in L'alimentation en forêt tropicale. Interactions bioculturelles et perspectives de développement, vol. II, C.M. Hladik, A. Hladik, H. Pagezy, O. Linares, G. Koppert et A. Froment (éd.), Unesco, Paris, p. 897-902.

IMAMURA Kikabu

1996, Prehistoric Japan. New perspectives on insular East Asia, University of Hawai'i Press, Honolulu.

LATHRAP Donald

1970, The upper Amazon, Thames and Hudson, Londres.

Le Pennec Jean-Luc, Geoffroy de Saulieu, Pablo Samaniego, Diego Jaya et Lydie Gailler 2013, « A devastating plinian eruption at Tungurahua volcano reveals Formative occupation at 1100 CAL BC in central Ecuador », Radiocarbon, 55 (2-3) (actes du 21st International Radiocarbon Conference, A.J.T. Jull et C. Hatté [éd.]), p. 1199-1214.

Meggers Betty J., Clifford Evans et Emilio Estrada

1965, Valdivia and Machalilla phases, Smisthonian Institution, Washington.

Neves Eduardo

2007, «El Formativo que nunca terminó: la larga historia de estabilidad en las ocupaciones humanas de la Amazonía central », Boletín de arqueología PUCP, 11, p. 117-142.

PAGÁn Jiménez Jaime R. et Stéphen Rostain

2014, «Uso de plantas económicas y rituales (medicinales o energizantes) en dos comunidades precolombinas de la Alta Amazonía ecuatoriana: Sangay (Huapula) y Colina Moravia (c. 400 a.C.-1200 d.C.) », in Antes de Orellana. Actas del 3er Encuentro internacional de arqueología amazónica, S. Rostain (éd.), IFEA/ FLACSO/Embajada de los EEUU, Quito, p. 313-322.

PORRAS Pedro

1987, Investigaciones arqueológicas a las faldas del Sangay. Tradición upano, Pontifica Universidad Católica del Ecuador, Quito.

Rostain Stéphen

1999, « Secuencia arqueológica en montículos del valle del Upano en la Amazonía ecuatoriana », Bulletin de l'Institut français d'études andines, 28 (1), p. 53-89.

2006, « Etnoarqueología de la casa huapula y jívaro », Bulletin de l’Institut français d'études andines, 35 (3) (numéro thématique « Avances de investigación en el Ecuador prehispánico », M. Guinea et J.-F. Bouchard [éd.]), p. 337-346. 
2010, « Cronología del valle del Upano, alta Amazonía ecuatoriana », Bulletin de l'Institut français d'études andines, 39 (3) (numéro thématique « Culturas y pueblos del Ecuador prehispánico », M. Guinea et J.-F. Bouchard [éd.]), p. 667-681.

2011, «Ethnoarchaeologogy of the Amazonian house: pre-Columbian and Jivaro continuity in Ecuador ", in Communities in contact. Essays in archaeology, ethnohistory et ethnography of the Amerindian circum-Caribbean, C.L. Hofman et A. van Duijvenbode (éd.), Sidestone Press, Leiden, p. 455-475.

2012, « Between Sierra and Selva: pre-Columbian landscapes in the upper Ecuadorian Amazonia », Quaternary international, 249 (numéro spécial « Human occupation of tropical rainforests », Norm Catto [éd.]), p. 31-42.

Rostain Stéphen et Geoffroy de SAulieu

2013, Antes. Arqueología de la Amazonía ecuatoriana, IFEA/IRD/IPGH, Quito.

2014, «El sol se levanta por el Este. Arqueología en la Amazonía ecuatoriana », INPC. Revista del patrimonio cultural del Ecuador, 5, p. 42-55.

Rostain Stéphen, Geoffroy de SAulieu et Emmanuel LÉzy

2014, «El alto Pastaza precolombino en el Ecuador: del mito a la arqueología », in Amazonía. Memorias de las conferencias magistrales del 3er Encuentro internacional de arqueología amazónica, S. Rostain (éd.), MCCTH/SENESCYT/3EIAA, Quito, p. 159-185.

Saulieu Geoffroy de, Stéphen Rostain et Jean-Luc Le Pennec

2014, «El Formativo del Alto Pastaza (Ecuador), entre arqueología y vulcanología », in Antes de Orellana. Actas del 3er Encuentro internacional de arqueología amazónica, S. Rostain (éd.), IFEA/FLACSO/Embajada de los EEUU, Quito, p. 199-205.

Valdez Francisco, Jean Guffroy, Geoffroy de Saulieu, Julio Hurtado et Alexandra YePez 2005, « Découverte d'un site cérémoniel formatif sur le versant oriental des Andes », Palévol, 4 (4), p. 369-374.

Villalba Marcello

1988, Cotocollao, una aldea formativa del valle de Quito, Museo del Banco Central (Miscelánea Antropológica Ecuatoriana, Serie monográfica, 2), Quito.

WIENER Charles

1882, «L'Amazone et les cordillères », in Le tour du monde, Librairie Hachette et Cie, Paris, p. 209-304.

ZARILLO Sonia

2012, Human adaptation, food production, and cultural interaction during the Formative period in highland Ecuador, thèse de doctorat en archéologie, University of Calgary, Calgary.

ZeIDLER James A.

1984, Social space in Valdivia society: community patterning and domestic structure at Real Alto, 3000-2000 B.C., thèse de doctorat en anthropologie, University of Illinois at Urbana-Champaign, Urbana.

2008, « The Ecuadorian Formative », in Handbook of South American archaeology, H. Silverman et W. Isbell (éd.), University of Illinois at Urbana-Champaign, Urbana, p. 459-488. 
